\title{
On Some Numerical Dissipation Schemes
}

\author{
R.C. Swanson \\ NASA Langley Research Center \\ Hampton, VA \\ email: swanson@tabdemo.larc.nasa.gov \\ R. Radespiel \\ DLR, Institute of Design Aerodynamics \\ Braunschweig, Germany \\ email: rolf.radespiel@dlr.de
}

E. Turkel

School of Mathematical Sciences

Sackler Faculty of Exact Sciences

Tel-Aviv University

Tel-Aviv, Israel

and ICASE, NASA Langley

email: turkel@math.tau.ac.il

\begin{abstract}
Several schemes for introducing an artificial dissipation into a central difference approximation to the Euler and Navier Stokes equations are considered. The focus of the paper is on the convective upwind and split pressure (CUSP) scheme, which is designed to support single interior point discrete shock waves. This scheme is analyzed and compared in detail with scalar dissipation and matrix dissipation (MATD) schemes. Resolution capability is determined by solving subsonic, transonic, and hypersonic flow problems. A finite-volume discretization and a multistage time-stepping scheme with multigrid are used to compute solutions to the flow equations. Numerical solutions are also compared with either theoretical solutions or experimental data. For transonic airfoil flows the best accuracy on coarse meshes for aerodynamic coefficients is obtained with a simple MATD scheme. The coarsegrid accuracy for the original CUSP scheme is improved by modifying the limiter function used with the scheme, giving comparable accuracy to that obtained with the MATD scheme. The modifications reduce the background dissipation and provide control over the regions where the scheme can become first order.
\end{abstract}




\section{Introduction}

Accuracy must be a primary consideration in the construction of any numerical scheme. In principle one would like to devise a discrete scheme with the minimum amount of artificial dissipation required for stability, as well as convergence in the case of a stationary solution. This usually means imposing the additional constraint that the order of the numerical dissipation is at least one order of magnitude smaller than the desired order of approximation. For general fluid dynamic computations the numerical scheme should be designed to have high accuracy in smooth regions of the flow field and high resolution at shock waves and contact discontinuities. According to Harten [3] such discrete formulations, where the accuracy away from discontinuities is at least second order, are called high resolution schemes. The design of these schemes for systems of conservation laws is generally based on theory developed for a scalar conservation law. As a consequence one cannot ensure that the properties of the scheme for the scalar equation are valid for the system. In addition, schemes that permit high definition of shock waves without oscillations are first order in the neighborhood of shocks. Concern naturally arises regarding contamination of the solution, especially in the case of viscous flows. For these reasons the properties and resolution capability of this class of schemes must be determined through numerical applications for a wide range of flow conditions.

High resolution schemes of particular interest for solving the compressible Euler and Navier-Stokes equations are those that allow shock capturing with a single interior point. In [6] Jameson presents two schemes with this property that are derived from two different forms of flux splitting. One scheme is designated a characteristic split formulation, and it employs the flux difference splitting and linearization technique of Roe [19]. With this scheme the diffusive flux depends on a flux Jacobian matrix. The other scheme is called the convective upwind and split pressure (CUSP) scheme. For this scheme the artificial diffusive flux vector associated with a given coordinate direction is expressed in terms of changes in the state and flux vectors. A somewhat limited number of inviscid and viscous computations have been performed to evaluate these schemes (see [6]-[7] and [27]-[28]).

We shall investigate and analyze the CUSP scheme, with emphasis on the HCUSP version which allows a solution with constant total enthalpy for steady flow. We discuss the shockcapturing behavior for various choices of the dissipation coefficients. We introduce a simple modification of the limiter function, which is generally used with the scheme, to control background dissipation, and thus global accuracy. Global accuracy is also improved by introducing parameters into the limiter function to augment control over the regions where the CUSP scheme can become first order. The CUSP scheme includes a contribution that is scaled according to the local velocity. If the velocity vanishes, as it does for viscous flows, and there is a high aspect ratio mesh, the dissipation in the streamwise direction (i.e., direction of long side of mesh cell) may not be adequate for convergence. A change in the velocity scaling factor based on aspect ratio is presented. The resolution capability of the HCUSP scheme is evaluated for subsonic, transonic, and hypersonic flow problems. A detailed comparison of the scheme with scalar and matrix dissipation schemes is performed. The scalar scheme is based on the dissipation model of Jameson, Schmidt, and Turkel [4]. 


\section{Dissipation}

A finite-volume approach is applied to discretize the fluid dynamic equations of motion. The computational domain is divided into quadrilateral cells, fixed in time, and for each cell the governing equations can be nondimensionalized and written in integral form as follows:

$$
\frac{\partial}{\partial t} \iint_{\Omega} w d x d y+\int_{\partial \Omega}(f d y-g d x)=\frac{\sqrt{\gamma} M}{R e} \int_{\partial \Omega}\left(f_{v} d y-g_{v} d x\right)
$$

where $\Omega$ is a generic cell (or cell area) with $\partial \Omega$ its boundary. In the scaling factor for the viscous terms on the right hand side of (2.1), the quantities $\gamma, M$, and $R e$ are the specific heat ratio, Mach number, and Reynolds number, respectively, with $M$ and $R e$ defined in terms of nominal conditions. Taking $w_{j, k}$ as the cell-averaged solution vector, equation (2.1) can be written in semi-discrete form as

$$
\frac{d}{d t}\left(\Omega_{j, k} w_{j, k}\right)+\mathcal{L} w_{j, k}=0
$$

where $\Omega_{j, k}$ is the area of the cell, and $\mathcal{L}$ is a spatial discretization operator defined by

$$
\mathcal{L}=\mathcal{L}_{C}+\mathcal{L}_{D}+\mathcal{L}_{A D}
$$

with the subscripts $C, D$, and $A D$ referring to convection, diffusion, and artificial dissipation. In order to simplify the description of the dissipation model, we consider the one-dimensional Euler equations of gas dynamics.

\subsection{Scalar Dissipation Model}

The scalar dissipation is based on the model introduced by Jameson, Schmidt, and Turkel [4]. This model defines a switching function based on a blending of the second and fourth differences. The term associated with the operator $\mathcal{L}_{A D}$ is expressed as

$$
\mathcal{L}_{A D} w_{j}=-\left(D^{2}-D^{4}\right) w_{j}=d_{j+1 / 2}-d_{j-1 / 2} .
$$

Then

$$
\begin{aligned}
& D^{2} w_{j}=\nabla\left[\left(\lambda_{j+1 / 2} \varepsilon_{j+1 / 2}^{(2)}\right) \Delta\right] w_{j}, \\
& D^{4} w_{j}=\nabla\left[\left(\lambda_{j+1 / 2} \varepsilon_{j+1 / 2}^{(4)}\right) \Delta \nabla \Delta\right] w_{j},
\end{aligned}
$$

where the index $j$ refers to a cell center, and the operators $\Delta$ and $\nabla$ are forward and backward difference operators. The variable scaling factor $\lambda$ is defined as

$$
\lambda_{j+1 / 2}=\frac{1}{2}\left[\lambda_{j}+\lambda_{j+1}\right],
$$

where $\lambda$ is the largest eigenvalue in absolute value (i.e., spectral radius) of the flux Jacobian matrix associated with the Euler equations. For example, in the $\xi$ and $\eta$ directions of generalized coordinates $(\xi, \eta)$,

$$
\lambda_{\xi}=\left|u y_{\eta}-v x_{\eta}\right|+c \sqrt{x_{\eta}^{2}+y_{\eta}^{2}},
$$




$$
\lambda_{\eta}=\left|u x_{\xi}-u y_{\xi}\right|+c \sqrt{x_{\xi}^{2}+y_{\xi}^{2}}
$$

The coefficients $\varepsilon^{(2)}$ and $\varepsilon^{(4)}$ use the pressure as a sensor for sharp gradients, and they are defined as

$$
\begin{aligned}
\varepsilon_{j+1 / 2}^{(2)} & =\kappa^{(2)} \max \left(\nu_{j-1}, \nu_{j}, \nu_{j+1}, \nu_{j+2}\right), \\
\nu_{j} & =\left|\frac{p_{j-1}-2 p_{j}+p_{j+1}}{p_{j-1}+2 p_{j}+p_{j+1}}\right| \\
\varepsilon_{j+1 / 2}^{(4)} & =\max \left[0,\left(\kappa^{(4)}-\varepsilon_{j+1 / 2}^{(2)}\right)\right]
\end{aligned}
$$

where typical values for the constants $\kappa^{(2)}$ and $\kappa^{(4)}$ are in the ranges $\frac{1}{4}$ to $\frac{1}{2}$ and $\frac{1}{64}$ to $\frac{1}{32}$, respectively. We shall refer to (2.4) together with (2.8) as the JST scheme and (2.8) alone as the JST switch. The switching function $\nu$ can be interpreted as a limiter, in the sense that it activates the second-difference contribution at extrema and switches off the fourth-difference term. Moreover, at shock waves the dissipation is first order, and a first-order upwind scheme is produced for a scalar equation. In smooth regions of the flow field the dissipation is third order.

Thus, we have two different dissipation mechanisms at work. The switch determines which one is active in any given region. For smooth flows, $\nu$ is small and the dissipation terms consists of a linear fourth difference that damps the high frequencies which the central difference scheme does not damp. This is useful for achieving a steady state and is not always necessary for time dependent problems [9]. In the neighborhood of large gradients in the pressure, $\nu$ becomes large and switches on the second-difference viscosity while simultaneously reducing the fourth-difference dissipation. This is mainly needed to introduce an entropy condition to reduce overshoots near discontinuities and choose the correct shock relationships. For subsonic steady state flow this can be turned off by choosing $\kappa^{(2)}=0$.

One possible extension of the scaling factor of (2.7) to multidimensions is isotropic. In two dimensions, with $(\xi, \eta)$ denoting arbitrary curvilinear coordinates, the scaling factor takes the form

$$
\lambda_{j+1 / 2, k}=\frac{1}{2}\left[\left(\lambda_{\xi}\right)_{j, k}+\left(\lambda_{\xi}\right)_{j+1, k}+\left(\lambda_{\eta}\right)_{j, k}+\left(\lambda_{\eta}\right)_{j+1, k}\right]
$$

Such a scaling is generally satisfactory for inviscid flow problems when typical inviscid flow meshes (i.e., cell aspect ratio $O(1)$ ) are used. This factor can cause excessive numerical dissipation in cases of meshes with high-aspect-ratio cells. Instead, the scaling factor is usually defined as

$$
\lambda_{j+1 / 2, k}=\frac{1}{2}\left[\left(\bar{\lambda}_{\xi}\right)_{j, k}+\left(\bar{\lambda}_{\xi}\right)_{j+1, k}\right]
$$

where

$$
\begin{aligned}
& \left(\bar{\lambda}_{\xi}\right)_{j, k}=\phi_{j, k}(r)\left(\lambda_{\xi}\right)_{j, k}, \\
& \phi_{j, k}(r)=2^{\zeta-1}\left(1+r_{j, k}^{\zeta}\right),
\end{aligned}
$$


$r$ is the ratio $\lambda_{\eta} / \lambda_{\xi}$, and the exponent $\zeta$ is defined by $0 \leq \zeta \leq 1$. If $\zeta=1$, the isotropic form of (2.9) is recovered. If $\zeta=0$, the scaling in a given direction simply depends on the eigenvalue associated with that direction. This scaling is sometimes called individual eigenvalue scaling (see [14], [21]). The exponent $\zeta$ is generally taken to be between $\frac{1}{2}$ and $\frac{2}{3}$. Thus, this dissipation scaling factor is between the isotropic and individual eigenvalue scaling factors. As demonstrated in [13] and [21], this factor produces a significant improvement in accuracy relative to the isotropic factor for high-aspect-ratio meshes, and it permits good convergence rates with a multigrid method.

Using the TVD concept, an alternative for the switch of (2.8) that is TVD for a scalar equation is introduced in [22]. In one dimension this switch is given by

$$
\nu_{j}=\frac{\left|p_{j+1}-2 p_{j}+p_{j-1}\right|}{\left|p_{j+1}-p_{j}\right|+\left|p_{j}-p_{j-1}\right|+\epsilon} .
$$

and choose $\kappa^{(2)}=\frac{1}{2}$. In practice we usually use a weaker form than $(2.12)$, for example,

$$
\nu_{j}=\frac{\left|p_{j+1}-2 p_{j}+p_{j-1}\right|}{(1-\omega) \mathcal{P}_{T V D}+\omega \mathcal{P}}
$$

where

$$
\begin{aligned}
\mathcal{P}_{T V D} & =\left|p_{j+1}-p_{j}\right|+\left|p_{j}-p_{j-1}\right|, \\
\mathcal{P} & =p_{j+1}+2 p_{j}+p_{j-1},
\end{aligned}
$$

and $0 \leq \omega \leq 1$. The TVD switch of $(2.12)$ is recovered when $\omega \ll 1$. Typically $\omega \sim 1 / 2$. In [23] this switch allowed the computation of flows with strong shock waves whereas the switch of (2.8) did not.

\subsection{Matrix-Valued Dissipation Model (MATD)}

Sharp resolution of shock waves without oscillations can be achieved by closely imitating an upwind scheme in the neighborhood of a shock wave. A key feature of upwind schemes is a matrix evaluation of the numerical dissipation. With this evaluation the dissipative terms of each discrete equation are scaled by the appropriate eigenvalues of the flux Jacobian matrix rather than by the spectral radius, as in the JST scheme. A matrix dissipation model can easily be constructed by starting with the JST formulation.

One can show [22] that the necessary modification of the JST scheme to produce a matrix dissipation model is the substitution of $|A|$ for the eigenvalue scaling factor $\lambda$ in (2.5) and (2.6). Since the Euler equations are a strongly hyperbolic system, the coefficient matrix can be diagonalized. Assume $Q A Q^{-1}=\Lambda$ (diagonal matrix). Then $|A|$ is defined as $|A|=Q^{-1}|\Lambda| Q$ and $|\Lambda|=\operatorname{diag}\left(\left|\lambda_{1}\right|,\left|\lambda_{2}\right|,\left|\lambda_{3}\right|\right)$, where $\lambda_{i}$ are the forward acoustic, backward acoustic, and convective eigenvalues. An efficient way of computing $|A|$ times a vector is presented in [22].

In practice one cannot choose $\lambda_{1}, \lambda_{2}, \lambda_{3}$ as the eigenvalues. Near stagnation points $\lambda_{3}$ approaches zero while near sonic lines $\lambda_{1}$ or $\lambda_{2}$ approaches zero. A zero artificial viscosity 
would create numerical difficulties. Hence, we limit these values as

$$
\begin{aligned}
& \left|\tilde{\lambda}_{1}\right|=\phi \max \left(\left|\lambda_{1}\right|, V_{n} \rho(A)\right), \quad\left|\tilde{\lambda}_{2}\right|=\phi \max \left(\left|\lambda_{2}\right|, V_{n} \rho(A)\right), \\
& \left|\tilde{\lambda}_{3}\right|=\phi \max \left(\left|\lambda_{3}\right|, V_{\ell} \rho(A)\right),
\end{aligned}
$$

where $\phi$ is defined by $(2.11), \rho(A)$ is the spectral radius of $\mathrm{A}$, and the linear eigenvalue $\lambda_{3}$ can be limited differently than the nonlinear eigenvalues. The parameters $V_{n}$ and $V_{\ell}$ have been determined numerically. Typical values are $V_{n}=0.25$ and $V_{\ell}=0.025$.

\subsection{CUSP Scheme}

In the previous sections we have described the use of an artificial viscosity based on either a scalar or matrix coefficient. Inspired by earlier work on flux-vector splitting [34] Liou and coworkers designed a scheme called Advection Upstream Splitting Method (AUSM) $[10,11,35]$. This method was later refined for large-scale 3 -D viscous computations in [17]. AUSM is based on a splitting of the flux function into convective and pressure contributions. In some sense, the pressure terms contribute to the acoustic waves while the velocity terms contribute to convective waves. Hence, it is reasonable that these flux terms be treated differently. Liou thus considers decompositions of the flux vector that are not based on a characteristic decomposition but on Mach number scaled contributions of the left and the right states to the interface flux. This decomposition has the disadvantage that it is more difficult to develop for other sets of equations compared with a characteristic decomposition. A similar type scheme called the Convective Upwind Split Pressure (CUSP) scheme was later introduced by Jameson [5] and subsequently modified by Tatsumi, Martinelli, and Jameson $[7,8,27,28]$. The CUSP scheme has some advantages over AUSM. First, one can consider the scheme as another type of artificial viscosity, since it is defined as a sum of the central flux average plus a dissipative flux. Hence, it can be readily used with a variety of time-stepping schemes (e.g., multistage, LU, implicit, etc.). Second, the CUSP formulation can be used in a straightforward manner with multistage schemes which do not evaluate the artificial dissipation fluxes at every stage, in order to reduce computational work. Hence, we shall only describe the CUSP version of this type of scheme.

\subsubsection{Definition of CUSP Scheme}

Previously, we introduced the scalar and matrix-valued viscosities by considering $d_{j+1 / 2}$ of the form

$$
d_{j+1 / 2}=\frac{1}{2} Q_{j+1 / 2}\left(w_{j+1}-w_{j}\right)
$$

The factor $\frac{1}{2}$ is introduced so that we get full upwinding when $Q_{j+1 / 2}=I$. We note that for the scheme to be positive, $Q$ must be sufficiently large. For the matrix viscosity we chose $Q=|A|$ (modified near zero eigenvalues) while for the scalar viscosity we chose $Q=\sigma(A) I$.

For the CUSP scheme we instead choose $d$ as a linear combination of $w$ and $f$. In one 
dimension we consider two choices for the state vector:

$$
\begin{aligned}
& w=(\rho \rho u \rho E)^{T} \\
& f=u\left(\begin{array}{c}
\rho \\
\rho u \\
\rho E
\end{array}\right)+\left(\begin{array}{c}
0 \\
p \\
u p
\end{array}\right)=u w+f_{p}
\end{aligned}
$$

and

$$
\begin{aligned}
w_{h} & =(\rho \rho u \rho H)^{T} \\
f & =u\left(\begin{array}{c}
\rho \\
\rho u \\
\rho H
\end{array}\right)+\left(\begin{array}{l}
0 \\
p \\
0
\end{array}\right)=u w_{h}+f_{p} .
\end{aligned}
$$

The first-order accurate CUSP scheme is defined as

$$
d_{j+1 / 2}=\frac{1}{2} \nu c\left(w_{j+1}-w_{j}\right)+\frac{\beta}{2}\left(f_{j+1}-f_{j}\right)
$$

The factor $c$ is included so that $\nu$ is dimensionless. We thus consider only scalar parameters instead of a matrix coefficient, but we have two free parameters, $\nu$ and $\beta$. The scheme is total enthalpy preserving if $w_{h}=(\rho \rho u \rho H)^{T}$ is chosen as the basis. This choice is denoted HCUSP by Jameson [7]. By using the arithmetic average, $\bar{u}=\frac{1}{2}\left(u_{j+1}+u_{j}\right)$, and the definition

$$
\alpha c=\nu c+\beta \bar{u}
$$

One can rearrange $(2.17)$ to obtain

$$
d_{j+1 / 2}=\frac{1}{2} \alpha c\left(w_{j+1}-w_{j}\right)+\frac{\beta}{2}\left(f_{p_{j+1}}-f_{p_{j}}\right)+\frac{\beta}{2} \bar{w}\left(u_{j+1}-u_{j}\right) .
$$

Introducing the Roe matrix $A_{R L}$, we have $f_{R}-f_{L}=A_{R L}\left(w_{R}-w_{L}\right)$. This relation is exact if $A_{R L}$ is computed from weighted averages of the left and the right states. That is,

$$
\begin{aligned}
u & =\frac{\sqrt{\rho_{R}} u_{R}+\sqrt{\rho_{L}} u_{L}}{\sqrt{\rho_{R}}+\sqrt{\rho_{L}}} \\
H & =\frac{\sqrt{\rho_{R}} H_{R}+\sqrt{\rho_{L}} H_{L}}{\sqrt{\rho_{R}}+\sqrt{\rho_{L}}} \\
\rho & =\sqrt{\rho_{R} \rho_{L}}
\end{aligned}
$$

Then the first-order dissipation is

$$
d_{j+1 / 2}=\frac{1}{2}\left(\beta A_{R L}+\nu c I\right)\left(w_{R}-w_{L}\right)
$$

We see from this formula that $d$ is a linear function of $A$. Recall that $|A|$ is a quadratic function of $A$, by the Cayley-Hamilton Theorem. Hence, it is not possible to bound $d$ by 


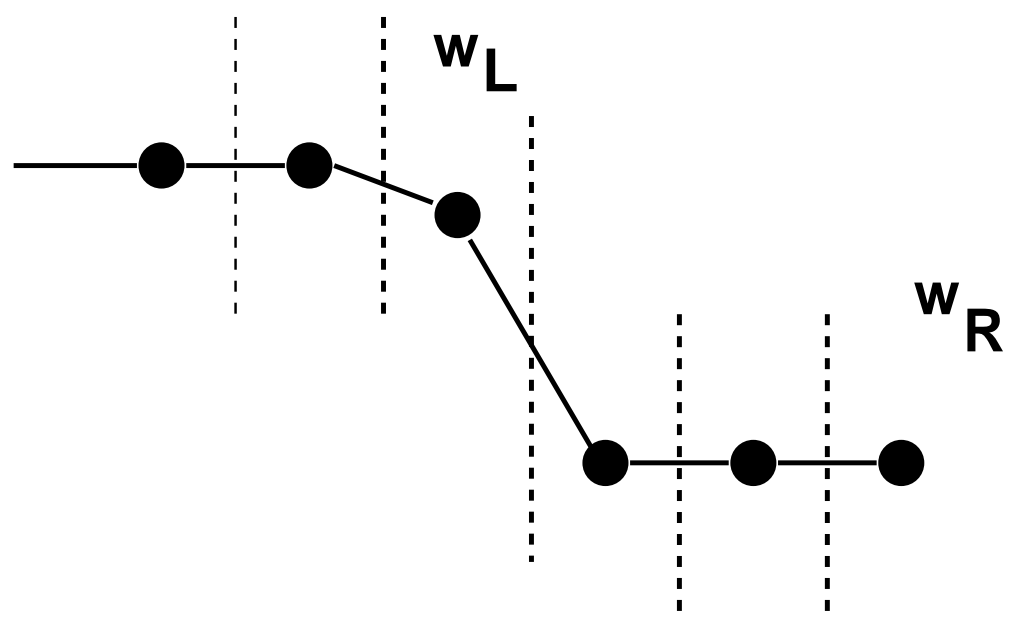

Figure 1: Conditions at shock wave.

$|A|$. Since $|A|$ is the minimum dissipation needed for a scheme to be positive [26], the CUSP scheme cannot be positive.

Remark. The parameters $\beta$ and $\nu$ will be defined later in this section. For these parameters the CUSP scheme is not positive at least for $M<1 / 2$. The concepts of $T V D$ and positivity were introduced primarily for the treatment of discontinuities. Thus, it is not clear theoretically if the loss of positivity for subsonic flow bounded away from the sonic line is important. For supersonic flow $(M \geq 1)$ the CUSP scheme is positive.

Assume that the subscript $L$ denotes the interior point inside the shock zone, $R$ is the state downstream of the shock, and the state $L R$ is subsonic (as depicted in Figure 1). Jameson [7] shows that the downstream point with the state $R$ is in equilibrium if

$$
f_{R}-f_{L}+\frac{\nu c}{1+\beta}\left(w_{R}-w_{L}\right)=0 .
$$

Substituting the Roe matrix for the difference in $f$ into (2.20) we get

$$
\left(A_{R L}+\frac{\nu c}{1+\beta} I\right)\left(w_{R}-w_{L}\right)=0 .
$$

Hence, $w_{R}-w_{L}$ is an eigenvector of $A_{R L}$, and $-(\nu c) /(1+\beta)$ is the corresponding eigenvalue. However, the eigenvalues of $A_{R L}$ are known to be $\lambda^{+}, \lambda^{-}$and $u$. If $\lambda$ is an eigenvalue of $A$, then using this formula for $\nu c$ in (2.19) we have

$$
d_{j+1 / 2}=\frac{1}{2}\left[-\lambda I+\beta\left(A_{R L}-\lambda I\right)\right]\left(w_{R}-w_{L}\right) .
$$

In order to have a positive diffusion when $u>0$, we require that $\lambda$ be negative (i.e., $\left.-(\nu c) /(1+\beta)=\lambda^{-}\right)$. Thus,

$$
\nu c=-(1+\beta) \lambda^{-}
$$


For $u<0$ we obtain similarly

$$
\nu c=(1-\beta) \lambda^{+}
$$

So, we have reduced our two free parameters to one free parameter by demanding a one point shock profile. More generally, Jameson shows that one obtains a shock profile with one interior point if the following two conditions hold:

1. When the flow is supersonic through the shock then one obtains a totally upwind flux.

2. The artificial dissipation $Q$ satisfies a generalized eigenvalue problem

$$
\left(A_{R L}-\alpha_{R A} Q_{R A}\right)\left(w_{R}-w_{A}\right)=0
$$

at the exit from the shock.

The second condition is satisfied by both the matrix viscosity and the CUSP scheme; however, the scalar viscosity does not satisfy the first condition. We again note that the positive condition $Q \geq|A|$ is satisfied by the scalar and matrix viscosities but not by the CUSP viscosity for all Mach numbers.

What remains to be done is to choose suitable functions for $\beta$ and $\nu c$ which satisfy the above requirements. Jameson's choice for $\beta$, which is based upon the eigenvalues corresponding to the acoustic waves, is given by

$$
\beta=\left\{\begin{array}{clc}
+\max \left(0, \frac{u+\lambda^{-}}{u-\lambda^{-}}\right) & \text {if } & 0 \leq M<1 \\
-\max \left(0, \frac{u+\lambda^{+}}{u-\lambda^{+}}\right) & \text {if } & -1<M<0 \\
\operatorname{sgn}(M) & \text { if } & |M| \geq 1
\end{array}\right.
$$

The cutoffs, $\beta \geq 0$ for $u>0$ and $\beta \leq 0$ for $u<0$, ensure that the pressure terms are discretized centrally for small Mach numbers. Shock capturing with one interior point is obtained by taking

$$
\nu c=\left\{\begin{array}{ccc}
|u| & \text { if } & \beta=0 \\
-(1+\beta) \lambda^{-} & \text {if } & \beta>0 \text { and } 0<M<1 \\
(1-\beta) \lambda^{+} & \text {if } & \beta<0 \text { and }-1<M<0 \\
0 & \text { if } & |M| \geq 1
\end{array}\right.
$$

The dissipation coefficients are to be computed from Roe-averaged quantities as in (2.18). They provide full upwinding for supersonic flow, $\beta=\operatorname{sgn}(M), \nu=0$. The choice of $\nu c=|u|$ for $\beta=0$ yields a continuous dissipation coefficient in the subsonic region, and it does not smear slip lines with $|u|$ close to zero. This makes the CUSP formulation attractive for viscous flow calculations with boundary layers. However, viscous flows are usually discretized by using cells with large aspect ratio. It is well known that this situation requires larger 
dissipation scaling in the direction of the long cell sides than given by $|u|$. We redefine the dissipation coefficients in the individual coordinate directions. For the $\xi$-direction we have

$$
\nu c_{\xi}=r^{+}\left\{\begin{array}{ccc}
\max \left(|u|, \delta c r^{-}\right) & \text {if } & \beta=0 \\
-(1+\beta) \lambda^{-} & \text {if } & \beta>0 \text { and } \\
& & 0<M<1 \\
(1-\beta) \lambda^{+} & \text {if } & \beta<0 \text { and } \\
& & -1<M<0 \\
0 & \text { if } & |M| \geq 1 .
\end{array}\right.
$$

where $r^{+}$and $r^{-}$are functions of the spectral radii in the $\xi$ and $\eta$ directions $\left(\lambda_{\xi}\right.$ and $\left.\lambda_{\eta}\right)$, and they are defined as follows:

$$
r=\left(\frac{\lambda_{\eta}}{\lambda_{\xi}}\right)^{\zeta}, \quad r^{+}=\max (r, 1), \quad r^{-}=\min (r, 1) .
$$

The dissipation coefficient in the $\eta$-direction is defined correspondingly.

\subsubsection{Simplified Scheme}

Several modifications of the CUSP scheme have been in use so far. Based upon the $w_{h}=$ $(\rho \rho u \rho H)^{T}$ system the dissipation coefficients presented in [7] and [28] are as follows:

$$
\begin{aligned}
& \alpha=\left\{\begin{array}{cll}
|M| & \text { if } & |M| \geq \epsilon \\
\frac{1}{2}\left(\epsilon+\frac{M^{2}}{\epsilon}\right) & \text { if } & |M|<\epsilon,
\end{array}\right. \\
& \beta=\left\{\begin{array}{clc}
+\max \left(0, \frac{u+\lambda^{-}}{u-\lambda^{-}}\right) & \text {if } & 0 \leq M<1 \\
-\max \left(0, \frac{u+\lambda^{+}}{u-\lambda^{+}}\right) & \text {if } & -1<M<0 \\
\operatorname{sgn}(M) & \text { if } & |M| \geq 1 .
\end{array}\right.
\end{aligned}
$$

This choice does not allow exact shock capturing because (2.21) and (2.22) are not satisfied. Furthermore, Roe averaging has been replaced by arithmetic averaging in [7] and $\lambda^{-}, \lambda^{+}$by $u-c, u+c$, respectively. This simplification saves a few square roots in the coding of the dissipative flux. Equation (2.25) is then

$$
\begin{aligned}
& \alpha=\left\{\begin{array}{cl}
|M| & \text { if }|M| \geq \epsilon \\
\frac{1}{2}\left(\epsilon+\frac{M^{2}}{\epsilon}\right) & \text { if }|M|<\epsilon,
\end{array}\right. \\
& \beta=\left\{\begin{array}{cl}
\max (0,2 M-1) & \text { if } \quad 0 \leq M<1 \\
\min (0,2 M+1) & \text { if }-1<M<0 \\
\operatorname{sgn}(M) & \text { if } \quad|M| \geq 1 .
\end{array}\right.
\end{aligned}
$$




\section{Higher Order Scheme}

Having determined $\nu c$ and $\beta$, we see from (2.17) that the scheme is completely defined in terms of $w$ and $f_{p}$. Formula (2.17), as given, is only first-order accurate, as it depends only on $d_{j+1 / 2}=w_{j+1}-w_{j}$, and so the complete artificial viscosity behaves like a second difference. The purpose of this section is to combine a first-order accurate CUSP scheme with a high-order dissipation.

Previously, we considered a combination of a low-order and high-order artificial viscosity based on the scalar (JST) switch of (2.8). This switch has the disadvantage that one quantity, the pressure, controls the shock sensor. Moreover, it forces all variables to be treated equal, even though some experience sharp changes through the discontinuity while others are continuous across the shock. The requirement to choose a particular flow variable for a switch can be eliminated. One can instead limit independently each dependent variable in each coordinate direction. Such a limiting allows the construction of a strictly upwind scheme for the one-dimensional Euler equations rather than just for a scalar equation.

In [5] Jameson constructed a family of limiter functions based on the function

$$
R(u, v)=1-\left|\frac{u-v}{|u|+|v|+\epsilon}\right|^{q},
$$

where $q$ is a positive number and $\epsilon$ has the dimensions of $u$. The parameter $\epsilon<<1$, and in this work it is taken to be $10^{-10}$. Note that $R(u, v) \approx 0$ whenever $u$ and $v$ have the opposite sign. Let $w$ be an element of the solution vector for the governing flow equations. Also, note that according to our previous theory [22] $R\left(\Delta w_{j+3 / 2}, \Delta w_{j-1 / 2}\right)$, where $\Delta w_{j+3 / 2}=w_{j+2}-w_{j+1}$, would be replaced by $\nu_{j+1 / 2}$, where $\nu_{j+1 / 2}$ is the maximum of $\nu_{j}$ over the nearest neighbors and $\nu$ is given by (2.12).

In the results section of this paper we will demonstrate that it can be beneficial to further control the regions where the limiter is applied. Hence, we generalize (3.1) to

$$
R(u, v)=1-\min \left(e_{v}, e_{p}, 1\right)\left|\frac{u-v}{|u|+|v|+\epsilon}\right|^{q},
$$

with

$$
\begin{aligned}
& e_{v}=\left\{\begin{array}{cc}
0 & \text { if } M \leq M_{\text {limit }} \\
5 M_{\text {limit }}^{-1}\left(M-M_{\text {limit }}\right) & \text { if } M>M_{\text {limit }},
\end{array}\right. \\
& e_{p}=\left\{\begin{array}{cc}
0 & \text { if } \nu \leq \nu_{\text {limit }} \\
2 \nu_{\text {limit }}^{-1}\left(\nu-\nu_{\text {limit }}\right) & \text { if } \nu>\nu_{\text {limit }} .
\end{array}\right.
\end{aligned}
$$

The control parameters are the contravariant Mach number $M$ and the pressure switch $\nu$, as given in (2.13). Thus, the limiter cannot produce a first-order scheme in regions where $M \leq M_{\text {limit }}$ or $\nu \leq \nu_{\text {limit }}$. With the introduction of $\min \left(e_{v}, e_{p}, 1\right)$ in $(3.2)$ the scheme is not very sensitive to the value of the exponent $q$ (typically, $q=1$ or $q=2$ ). 
Define the limiter function $L(u, v)$ by

$$
L(u, v)=R(u, v) \frac{u+v}{2} .
$$

At the mesh cell interface $j+1 / 2$, we define the left and right states for each dependent variable as

$$
\begin{aligned}
& w_{L}=w_{j}+\frac{1}{2} L\left(\Delta w_{j+3 / 2}, \Delta w_{j-1 / 2}\right), \\
& w_{R}=w_{j+1}-\frac{1}{2} L\left(\Delta w_{j+3 / 2}, \Delta w_{j-1 / 2}\right),
\end{aligned}
$$

and so

$$
w_{R}-w_{L}=\Delta w_{j+1 / 2}-L\left(\Delta w_{j+3 / 2}, \Delta w_{j-1 / 2}\right) .
$$

For the artificial viscosity all differences will be based on $w_{R}-w_{L}$. In the neighborhood of shock waves $R(u, v)$ and hence $L(u, v)$ are close to zero. Moreover, $w_{R}-w_{L}=\Delta w_{j+1 / 2}$, resulting in a first-order scheme for the artificial viscosity. For smooth flow $R(u, v)=1$, and $L(u, v)=(u+v) / 2$. Hence, in a smooth region

$$
\begin{aligned}
w_{R}-w_{L}= & \Delta w_{j+1 / 2}-L\left(\Delta w_{j+3 / 2}, \Delta w_{j-1 / 2}\right) \\
& \simeq \Delta w_{j+1 / 2}-\frac{\Delta w_{j+3 / 2}+\Delta w_{j-1 / 2}}{2} \\
& =-\frac{1}{2} \Delta^{3} w_{j+1 / 2} .
\end{aligned}
$$

Thus, in the smooth regions $w_{R}-w_{L}$ behaves as a third difference, while in the vicinity of shock waves it behaves as a first difference. Consequently, (3.5) has similar properties to the JST scheme. One can obtain the relationship between (3.5) and the JST scheme by defining the diffusive flux $d_{j+1 / 2}$ as

$$
d_{j+1 / 2}=\alpha_{j+1 / 2}\left(w_{R}-w_{L}\right) \quad, \quad \alpha_{j+1 / 2}=\kappa^{(2)} \lambda_{j+1 / 2},
$$

where $\kappa^{(2)}$ is a parameter, and $\lambda$ is the spectral radius of the associated flux Jacobian matrix.

One difference between the JST scheme and (3.5) involves the parameters $\kappa^{(2)}$ and $\kappa^{(4)}$ for the second and fourth differences, respectively. Both $\kappa^{(2)}$ and $\kappa^{(4)}$ are free parameters in the JST scheme. As seen from (3.6) and (3.7) these parameters are automatically chosen as $\kappa^{(2)}$ and $\frac{1}{2} \kappa^{(2)}$ with (3.5). Furthermore, for the matrix viscosity (see Section 2.2) and the CUSP scheme (described in Section 2.3) $\kappa^{(2)}=\frac{1}{2}$, and so we no longer have any free parameters. The coefficient of the second difference is chosen as $\frac{1}{2}$ so that the scheme is fully upwind for supersonic flows. However, the fourth-difference viscosity is introduced only to accelerate the convergence to a steady state by eliminating the decoupling of the odd and even points. Hence, we wish $\kappa^{(4)}$ to be as small as possible for accuracy while still achieving a good convergence rate. It does not seem reasonable to connect the two components of the artificial viscosity. In section 4 we will compare the magnitude of the scalar viscosity and the CUSP scheme. 
One can generalize the limiter function of (3.3) by reintroducing a free parameter that essentially governs the level of the third-order viscosity in the smooth regions. The resulting scheme has the disadvantage that a free parameter must be chosen; however, it has the advantages of greater flexibility and increased accuracy. We now define a new $L$ as

$$
L(u, v, w)=R(u, w) \cdot\left[\left(1-4 \kappa^{(4)}\right) v+4 \kappa^{(4)} \frac{u+w}{2}\right],
$$

where left and right state values are determined by

$$
\begin{aligned}
w_{L} & =w_{j}+\frac{1}{2} L\left(\Delta w_{j+3 / 2}, \Delta w_{j+1 / 2}, \Delta w_{j-1 / 2}\right), \\
w_{R} & =w_{j+1}-\frac{1}{2} L\left(\Delta w_{j+3 / 2}, \Delta w_{j+1 / 2}, \Delta w_{j-1 / 2}\right) .
\end{aligned}
$$

When $\kappa^{(4)}=\frac{1}{4}$, the $L$ of $(3.8)$ reduces to the original $L$ of $(3.3)$. At shock waves $R(u, w) \approx 0$, and we again have $w_{R}-w_{L}=\Delta w_{j+1 / 2}$. For smooth regions of the flow field we have $w_{R}-w_{L}=-2 \kappa^{(4)} \Delta^{3} w_{j+1 / 2}$.

One difficulty with (3.1), and indeed with any TVD switch, is that it limits the differences near minima and maxima independent of the amplitude of the function. Hence, in the far field where the solution is almost uniform the low-order scheme is activated by small noise levels. Since this occurs randomly it frequently prevents the convergence of the residual beyond three or four orders of magnitude. The use of (3.2) eliminates this difficulty.

The extrapolation technique of (3.8) can be used with (2.17) to get the first difference to higher order accuracy. Then, the states corresponding to higher order accuracy are obtained in a way similar to van Leer's MUSCL approach [34]. To impose monotonicity one can apply the limiter discussed in this section. For example, we can replace (2.17) by

$$
d_{j+1 / 2}=\frac{\nu c}{2}\left(w_{R}-w_{L}\right)+\frac{\beta}{2}\left[f_{p}\left(w_{R}\right)-f_{p}\left(w_{L}\right)\right]
$$

where $w_{R}, w_{L}$ are given by (3.4). This procedure was followed throughout the numerical examples presented in Section 7. Application of (3.4) to the $w_{h}=(\rho \rho u \rho H)^{T}$ variables still allows total enthalpy to be preserved in the higher order scheme. When a multigrid algorithm is used to solve the governing flow equations, the higher order scheme is applied only on the finest mesh, and the lower order scheme is applied on the coarser meshes. 


\section{Analysis of CUSP Scheme}

The eigenvalues of $\beta A_{R L}+\nu c I$ (see 2.19) are $\mu_{1} c, \mu_{2} c$, and $\mu_{3} c$. Using the simplifications of (2.26) the eigenvalues are:

$$
\begin{aligned}
& \mu_{1}=|M|, \\
& \mu_{2}=\left\{\begin{array}{clc}
|M| & \text { if } & |M|<\frac{1}{2} \\
\alpha+\beta & \text { if } & \frac{1}{2} \leq M \leq 1 \\
|M+1| & \text { if } & |M|>1,
\end{array}\right. \\
& \mu_{3}=\left\{\begin{array}{ccc}
|M| & \text { if } & |M|<\frac{1}{2} \\
\alpha-\beta & \text { if } & \frac{1}{2} \leq M \leq 1 \\
|M-1| & \text { if } & |M|>1
\end{array}\right.
\end{aligned}
$$

We note that for $|M|<\frac{1}{2}$ all three eigenvalues of the artificial viscosity are equal, and so we have the equivalent of a scalar viscosity. The scalar viscosity now scales with $|M| c$ rather than $(|M|+1) c$ as in the JST scalar viscosity. This is more similar to the case of preconditioning where all the eigenvalues are approximately $|M| c$ for low speed flow. Hence, we expect that the CUSP dissipation should work properly for very low Mach numbers provided the central flux terms are augmented by a suitable preconditioning matrix.

In the subsonic range where $\beta=0$, all of the versions of the CUSP scheme do not satisfy (2.21) and (2.22), which are necessary for shock capturing. Thus the cell-face Mach numbers in the shock structure have to be larger than about 0.5 in order to avoid post-shock oscillations. The motivation to design $\nu c=|u|$ when $\beta=0$ has already been discussed. However, the choice of the function for $\beta$, as given in (2.23), is not necessarily optimal. For example, $\beta=\max \left(0,\left(u+\frac{1}{2} \lambda^{-}\right) /\left(u-\lambda^{-}\right)\right)$would allow shock capturing for Mach numbers down to about $1 / 3$, but the subsonic dissipation would be twice as large, $\nu c=2|u|$ for $\beta=0$. Nevertheless, our own experience gained from a number of numerical applications suggests that there is no need for further modifications of $\beta$.

It is rather difficult to compare the effect of the parameter $\kappa^{(4)}$ of the JST and the CUSP schemes, since these schemes also include eigenvalue information which is not the same in the two schemes. To isolate the effect we consider a low Mach number flow with preconditioning (see [31] for details). Now both switches are based on the convective eigenvalue u. A typical value for the $J S T$ scheme is $\kappa^{(4)}=\frac{1}{32}$. However, for an aspect ratio of one the Martinelli scaling [13] adds another factor of two. The parameter $\alpha=0$ in the preconditioning adds an additional factor of approximately 2.6. Hence, the effective constant multiplying the fourth difference is about $\frac{5}{32}$, which is somewhat smaller than the $\frac{1}{4}$ used with the original CUSP scheme. For transonic flows it is more difficult to compare the levels of dissipation. However, it seems that the original CUSP scheme yields too high a viscosity level and so the $\kappa^{(4)}$ introduced in (3.8) should be reduced to less than $\frac{1}{4}$. Numerical computations demonstrate the improved accuracy (though slower convergence) for standard transonic turbulent flows when $\kappa^{(4)}$ is reduced. 


\section{Low Speed Preconditioning}

For low Mach numbers standard algorithms converge to a steady state very slowly because of the disparity between the convective and acoustic eigenvalues. Furthermore, it is also found that most schemes give very poor results for these low Mach number flows, even when a steady state is achieved ([30]-[32]). One way to overcome these difficulties is to precondition the equations by multiplying the time derivative terms by a matrix $\mathbf{P}^{-1}$. If $\mathbf{P}$ is appropriately chosen, then one can reduce the disparity of the wave speeds. In this section preconditioning is applied to the different dissipation schemes. Details of preconditioning techniques are given in $[31,30]$.

To simplify the presentation we shall only consider a one-dimensional system. The extension to multidimensions is straightforward. Consider the system of equations

$$
\frac{\partial w}{\partial t}+\frac{\partial f}{\partial x}=0
$$

We replace this by the preconditioned system

$$
\mathbf{P}^{-1} \frac{\partial w}{\partial t}+\frac{\partial f}{\partial x}=0
$$

or in quasi-linear form

$$
\mathbf{P}^{-1} \frac{\partial w}{\partial t}+A \frac{\partial w}{\partial x}=0
$$

where $A=\partial f / \partial w$. Introducing an artificial viscosity in conservation form, we get

$$
\begin{aligned}
\mathbf{P}^{-1} \frac{\partial w}{\partial t}+\frac{D_{0} f}{2 \Delta x}=\frac{1}{\Delta x} \nabla\left[\varepsilon^{(2)} \mathbf{P}^{-1} F(\mathbf{P} A) \Delta w\right]=\frac{d_{j+1 / 2}-d_{j-1 / 2}}{\Delta x} \\
\frac{\partial w}{\partial t}+\mathbf{P} \frac{D_{0} f}{2 \Delta x}=\frac{\mathbf{P}}{\Delta x} \nabla\left[\varepsilon^{(2)} \mathbf{P}^{-1} F(\mathbf{P} A) \Delta w\right]=\mathbf{P} \frac{d_{j+1 / 2}-d_{j-1 / 2}}{\Delta x}
\end{aligned}
$$

where $D_{0}$ denotes a central difference, $\nabla$ is a backward difference, and $\Delta$ is a forward difference operator.

We first consider the matrix-valued viscosity, and thus $F(\mathbf{P} A)=|\mathbf{P} A|$. The artificial viscosity is

$$
\begin{aligned}
d_{j+1 / 2} & =\varepsilon_{j+1 / 2}^{(2)} \mathbf{P}_{j+1 / 2}^{-1}\left|(\mathbf{P} A)_{j+1 / 2}\right|\left(w_{j+1}-w_{j}\right) \\
\mathbf{P}_{j} d_{j+1 / 2} & =\varepsilon_{j+1 / 2}^{(2)} \mathbf{P}_{j} \mathbf{P}_{j+1 / 2}^{-1}\left|(\mathbf{P} A)_{j+1 / 2}\right|\left(w_{j+1}-w_{j}\right) .
\end{aligned}
$$

When $\mathbf{P} A$ has only three distinct eigenvalues, then by the Cayley-Hamilton theorem $|\mathbf{P} A|=$ $\alpha_{0} I+\alpha_{1} P A+\alpha_{2}(P A)^{2}$, where the coefficients $\alpha_{i}$ depend on the eigenvalues of $\mathbf{P} A$. So

$$
\mathbf{P}^{-1}|\mathbf{P} A|=\alpha_{0} \mathbf{P}^{-1}+\alpha_{1} A+\alpha_{2} \mathbf{P} A^{2}
$$

We next consider the CUSP artificial viscosity. The artificial viscosity term is given by $\alpha_{0} \Delta w+\alpha_{1} \Delta f \sim \alpha_{0} \Delta w+\alpha_{1} A \Delta w$ with the appropriate coefficients $\alpha_{i}$ for the CUSP scheme. 
This has the same form as the matrix-valued artificial viscosity without the quadratic term, and so by the identical reasoning we get

$$
\begin{aligned}
d_{j+1 / 2} & =\frac{1}{2} \nu c \mathbf{P}_{j+1 / 2}^{-1}\left(w_{j+1}-w_{j}\right)+\frac{\beta}{2}\left(f_{j+1}-f_{j}\right) \\
\mathbf{P}_{j} d_{j+1 / 2} & =\frac{1}{2} \nu c \mathbf{P}_{j} \mathbf{P}_{j+1 / 2}^{-1}\left(w_{j+1}-w_{j}\right)+\frac{\beta}{2} \mathbf{P}_{j}\left(f_{j+1}-f_{j}\right)
\end{aligned}
$$

(compare with (2.17)). In theory the parameters $\nu$ and $\beta$ should depend on the eigenvalues of $\mathbf{P} A$ rather than $A$ and so are not the same as the nonpreconditioned version of CUSP. However, in order to not interfere with the shock properties of the CUSP scheme we turn off the preconditioning for $M>1 / 2$. Hence, the relationship (2.21) is still valid. The parameter $\beta$ would then be chosen by (2.23) where the eigenvalues $\lambda^{+}, \lambda^{-}$should account for the preconditioning. In addition, for $|M|<1 / 2$ the CUSP scheme reduces to a scalar viscosity proportional to the convective velocity which is appropriate for preconditioning. Hence, it is reasonable to use the same parameters $\nu$ and $\beta$ for the preconditioned CUSP as given by (2.21) based on the original eigenvalues or one of the simplifications previously discussed. The advantages of combining the CUSP scheme with preconditioning are shown in Section 7. Additional results with the preconditioned CUSP scheme are presented in [33].

\section{Summary}

The central difference scheme requires an artificial viscosity in order to both prevent oscillations near shocks and damp high frequencies, enabling the iteration procedure to reach a steady state. In the Jameson, Schmidt, Turkel (JST) formulation these artificial viscosities are provided by second and fourth differences of the variables with a scalar coefficient included. This scalar coefficient depends on the largest eigenvalue (in each direction) to scale the size of the viscosity. In addition, the coefficient depends on the second difference of the pressure to sense shocks. In the neighborhood of shocks the fourth difference is turned off while the second difference prevents overshoots. In smooth regions of the flow the second difference (which leads to first-order accuracy) is minimal while the fourth difference damps the high-frequency errors.

This technique works quite well for transonic flow and was the main approach for many years. With the increasing popularity of upwind schemes it was seen that this scheme is less accurate than upwind schemes, especially on coarse meshes (see e.g., [1]). This led to the introduction of a matrix-valued coefficient in the artificial viscosity (dissipation) that mimics the effects of an upwind scheme, but within the context of a central difference scheme with an artificial dissipation coupled with a multistage time advancement. Later Jameson introduced the CUSP scheme, which is in between the matrix dissipation and the scalar dissipation schemes. With the CUSP scheme the dissipation is a function of the Mach number and becomes fully upwind in supersonic regions similar to the matrix dissipation. However, it avoids the need for a full-matrix coefficient while still obtaining one point shock profiles. The CUSP scheme is more expensive than the matrix-viscosity method, since it uses extrapolation for each flow variable and limiting depending on the variable to achieve second-order accuracy, 
The idea of using the Mach number to mimic fully upwind methods has been useful in other applications besides the artificial viscosity. One can use the Mach number to adjust the parameters of the residual smoothing so that it becomes fully upwind in supersonic regions. Similarly one can construct a multigrid method with weighting factors depending on the Mach number such that it becomes fully upwind in supersonic regions. We label this

\section{Poor Man's Upwinding}

\section{Advantages:}

- Cheap

- Fully upwind in supersonic flow

\section{Disadvantage:}

- Not fully upwind for subsonic flow

\section{Applications}

- Residual smoothing

- CUSP scheme

- Multigrid

In the opposite direction the Mach number can be used to construct a preconditioning that is useful in low Mach number regions. Then the Mach number can be used to turn off the preconditioning in supersonic regions.

\section{$7 \quad$ Numerical Results}

In the numerical applications presented here we assess the accuracy and shock capturing capabilities of the CUSP scheme. Since the version of the CUSP scheme that we consider is expressed in terms of the total enthalpy $H$ and is $H$ preserving for inviscid flows, it is usually called the HCUSP scheme. Comparisons are made between the HCUSP and MATD schemes. The commonly used scalar dissipation scheme is also included in some of the comparisons. In so doing one can clearly see the superiority of the high-resolution HCUSP and MATD schemes on even relatively coarse meshes (i.e., 8 cells in the boundary layer of a viscous flow). The flow problems considered in the evaluation of these numerical diffusion schemes include the following: 1) Inviscid flow over airfoils, 2) laminar flow over a flat plate, 3) turbulent flow over an airfoil, 4) inviscid and viscous hypersonic flow over a 2-D wedge. The computational effort and convergence behavior in computing these solutions are given. In all cases a five-stage Runge-Kutta scheme in conjunction with the convergence acceleration techniques of local time stepping, implicit residual smoothing, and multigrid was used. 


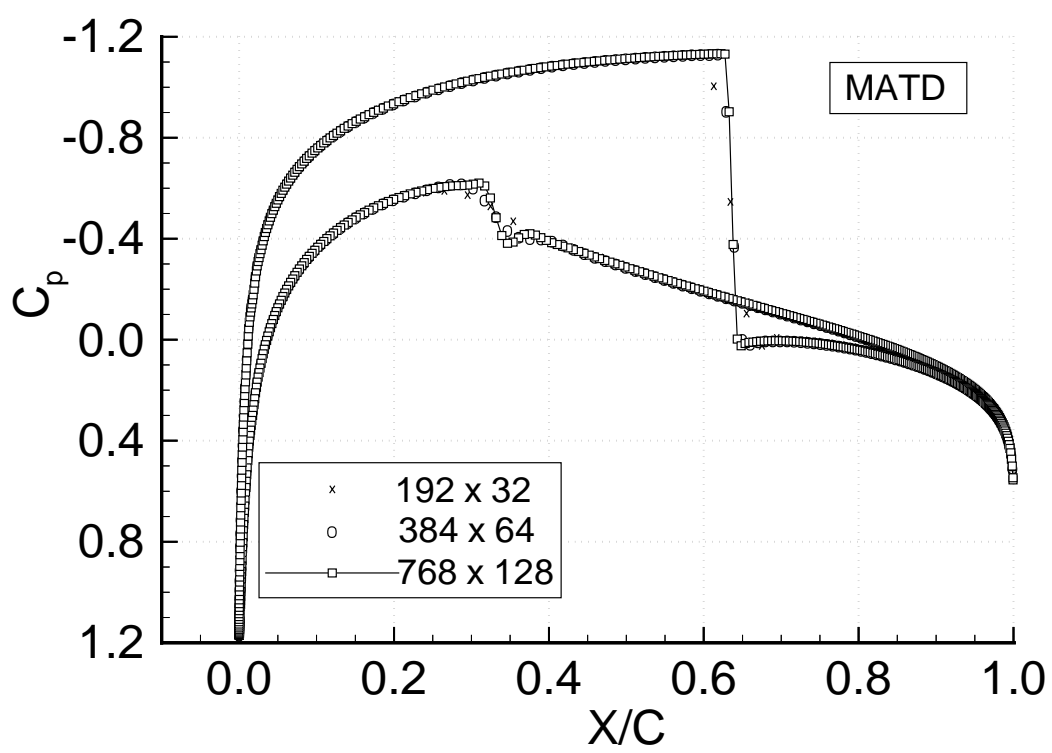

Figure 2a: Inviscid pressure distributions computed with MATD scheme (NACA 0012 airfoil, $M=0.80, \alpha=1.25^{\circ}$ ).

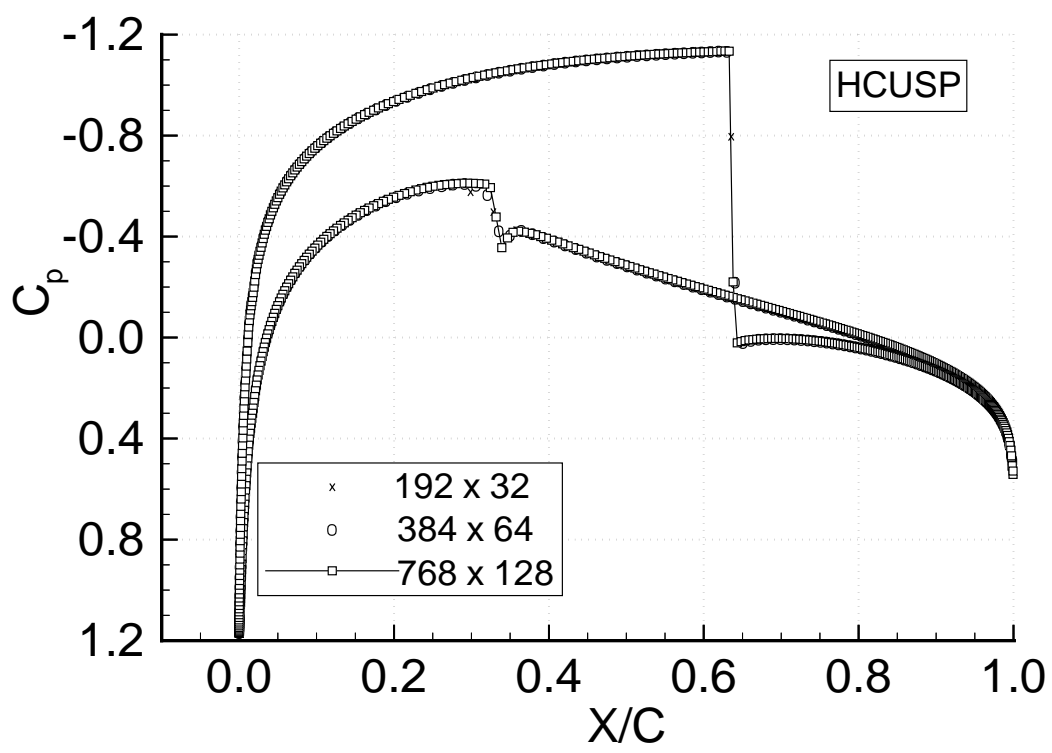

Figure 2b: Inviscid pressure distributions computed with HCUSP scheme (NACA 0012 airfoil, $M=0.80, \alpha=1.25^{\circ}$ ). 


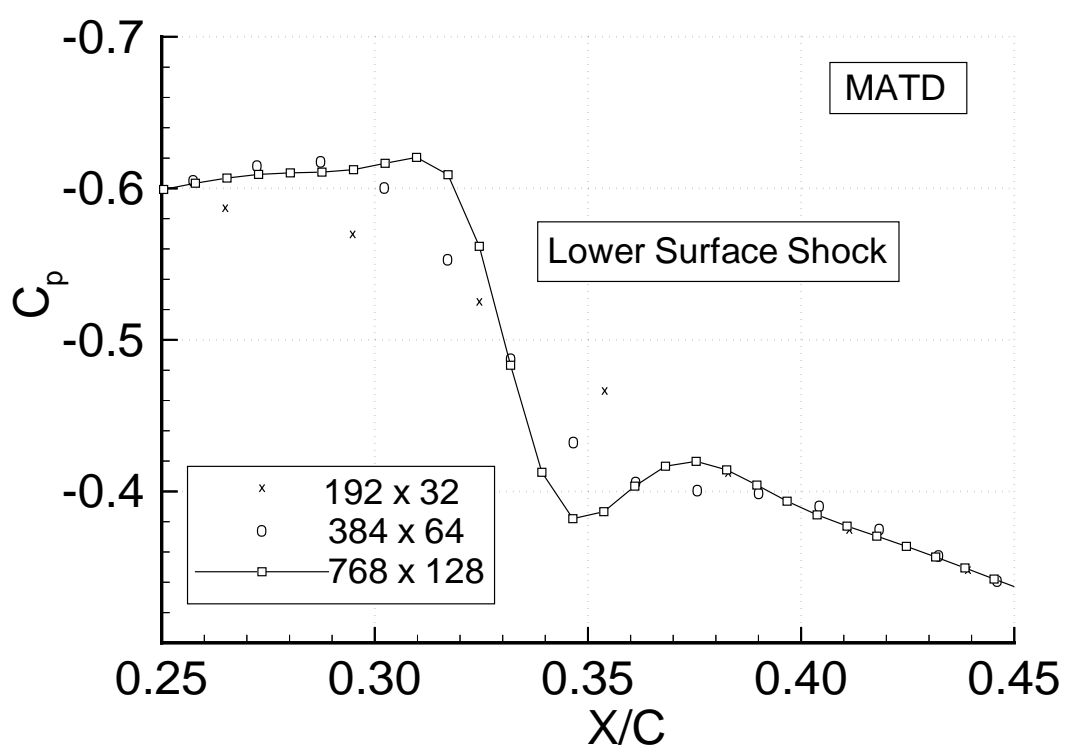

Figure 3a: MATD solutions near lower surface shock (NACA 0012 airfoil, $M=0.80, \alpha=$ $1.25^{\circ}$ ).

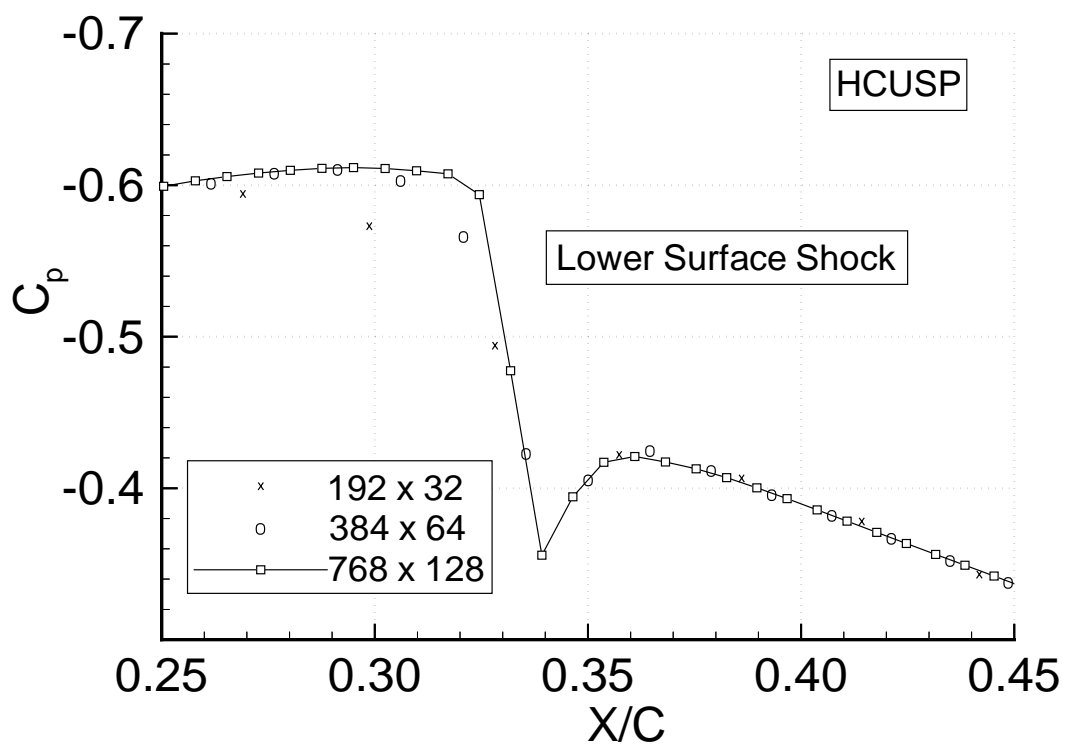

Figure 3b: HCUSP solutions near lower surface shock (NACA 0012 airfoil, $M=0.80$, $\left.\alpha=1.25^{\circ}\right)$. 


\begin{tabular}{|c|c|c|c|}
\hline $\begin{array}{c}\text { Dissipation } \\
\text { Scheme }\end{array}$ & Grid & $C_{L}$ & $C_{D}$ \\
\hline MATD & $192 \times 32$ & 0.3521 & 0.02249 \\
& $384 \times 64$ & 0.3550 & 0.02256 \\
& $768 \times 128$ & 0.3552 & 0.02256 \\
\hline HCUSP & $192 \times 32$ & 0.3667 & 0.02419 \\
& $384 \times 64$ & 0.3610 & 0.02310 \\
& $768 \times 128$ & 0.3582 & 0.02278 \\
\hline HCUSP & $192 \times 32$ & 0.3639 & 0.02297 \\
(modified) & $384 \times 64$ & 0.3592 & 0.02279 \\
& $768 \times 128$ & 0.3563 & 0.02269 \\
\hline
\end{tabular}

Table I: Lift and drag coefficients for inviscid flow over NACA 0012 airfoil $\left(M_{\infty}=0.80\right.$, $\alpha=1.25^{\circ}$.

The first case is similar to the application published in [7]. Results obtained with the MATD and HCUSP schemes for inviscid transonic flow over the NACA 0012 airfoil are compared in Figures 2 and 3. The free-stream Mach number for this case is 0.8 and the angle of attack is $1.25^{\circ}$. Solutions were computed on three successively finer C-topology meshes. The coarsest mesh contained $192 \times 32$ cells, with 160 cells on the airfoil, and for each sequential mesh the number of cells in each coordinate direction was doubled. The principal differences between the solutions occur at the shock waves. Since the MATD scheme uses a pressure switch for all the flow equations, it cannot capture a shock with a single interior point. It requires three interior points. Nevertheless, the resolution of the stronger upper surface shock is nearly the same for both the MATD and HCUSP schemes on the $384 \times 64$ and $768 \times 128$ meshes. With the MATD formulation there is some smearing on the $192 \times 32$ mesh. As is clearly evident in Figure 3 the HCUSP scheme allows a sharp definition of the weak lower surface shock and the Zierep singularity that immediately follows. The aerodynamic coefficients calculated with the MATD, original HCUSP (with limiter of (3.1)), and modified HCUSP (with limiter of (3.2)) schemes are presented in Table I. The coefficients computed with the MATD scheme on the $384 \times 64$ mesh essentially agree with those for the finest grid. The lift coefficients determined with the original and modified HCUSP schemes on the corresponding meshes are slightly higher, with the finest grid values approaching those obtained with the MATD scheme. Drag coefficients obtained with the modified HCUSP scheme are in closer agreement with those obtained with the MATD scheme, especially on the coarsest grid. Later, in the discussion viscous airfoil flow results we will show the behavior of the two forms of the limiter in the flow field.

As an initial evaluation of the dissipation schemes for viscous flows we consider low-speed $\left(M_{\infty}=0.15\right)$ flow over a flat plate at zero incidence. For this flow the Reynolds number per unit length is $10^{5}$. The computational domain is a rectangle. With respect to the leading 
edge of the plate, the domain extends two plate lengths upstream and one plate length downstream. The upper boundary is four plate lengths above the plate. Solutions were computed on the same domain and grids used in [28]. Starting with the finest mesh, coarser meshes were determined by successively eliminating every other mesh line. The finest grid consists of $512 \times 128$ cells, with 384 cells on the plate. In the direction $y$ normal to the plate the grid is spaced uniformly in the boundary-layer coordinate $\eta\left(\eta=y / R e_{x}^{1 / 2}\right)$, where $x$ is the coordinate parallel to the surface, and $R e_{x}$ is the Reynolds number based on distance from the leading edge of the plate). Thus, there is constant resolution of the boundary layer at each location along the plate. Outside the boundary layer the grid is stretched exponentially. In order to resolve the region in the vicinity of the stagnation point, the grid is clustered at the leading edge of the plate. At the surface of the plate no-slip and adiabatic boundary conditions are enforced. Along the boundary upstream of the leading edge, a symmetry condition is applied. Characteristic type boundary conditions are used at the upstream, downstream, and upper boundaries.

A comparison of the velocity profile at $X / L=0.82$ computed with the scalar, matrix, and HCUSP dissipation forms is displayed in Figure 4. Even with just eight points in the boundary layer $(64 \times 16$ grid $)$ the MATD and $H C U S P$ schemes nearly replicate the Blasius solution. As demonstrated in [1] scalar dissipation can produce serious contamination. With the scalar dissipation, more than 32 points are required in the boundary layer to obtain a grid converged solution. For the MATD and HCUSP schemes the variation of the errors (relative to the Blasius solution) in the calculated skin friction, displacement thickness, and momentum thickness are shown in Figures 5a and 5b. The standard definitions given in [20] are used for these boundary-layer quantities. The errors in all the boundary-layer parameters are quite similar for the high-resolution schemes. This is not surprising since both schemes have a scaling factor that vanishes as the surface is approached.

Transonic flow over the RAE 2822 airfoil is the next test case. The free-stream Mach number is 0.73 , the angle of attack is $2.79^{\circ}$, and the Reynolds number based on the airfoil chord is $6.5 \times 10^{6}$. Transition of the flow from laminar to turbulent is fixed at the $3 \%$ chord location. The C-type grids used in the computations are as follows: (1) $160 \times 32$ with 128 cells on the airfoil, (2) $320 \times 64$ with 256 cells on the airfoil, and (3) $640 \times 128$ with 512 cells on the airfoil. In order to determine the effect of further mesh refinement a calculation was performed with the MATD scheme on a $1280 \times 256$ grid. As in the flat-plate case, each successively coarser grid was generated by eliminating every other mesh line in both coordinate directions of the finer mesh. The outer boundary is located 20 chords from the airfoil. The normal spacing at the surface of the $640 \times 128$ mesh is $7.5 \times 10^{-6}$ chords. At the leading and trailing edges of the airfoil the mesh is clustered, giving tangential spacings of $1.17 \times 10^{-3}$ and $1.86 \times 10^{-3}$ chords, respectively. These critical mesh-defining spacings are roughly doubled with each mesh coarsening.

In Figure 6 the pressure $\left(C_{p}\right)$ and surface skin-friction $\left(C_{f}\right)$ distributions computed with the different dissipation schemes for the $160 \times 32$ mesh described are shown, along with the experimental data of [2]. As in the inviscid cases the primary differences in the solutions occur at the shock wave. Both the scalar dissipation (SCALAR) and HCUSP schemes produce a solution with the shock too far upstream. This is an unexpected result for the HCUSP scheme. The acceleration of the flow upstream of the shock is underpredicted relative to the 

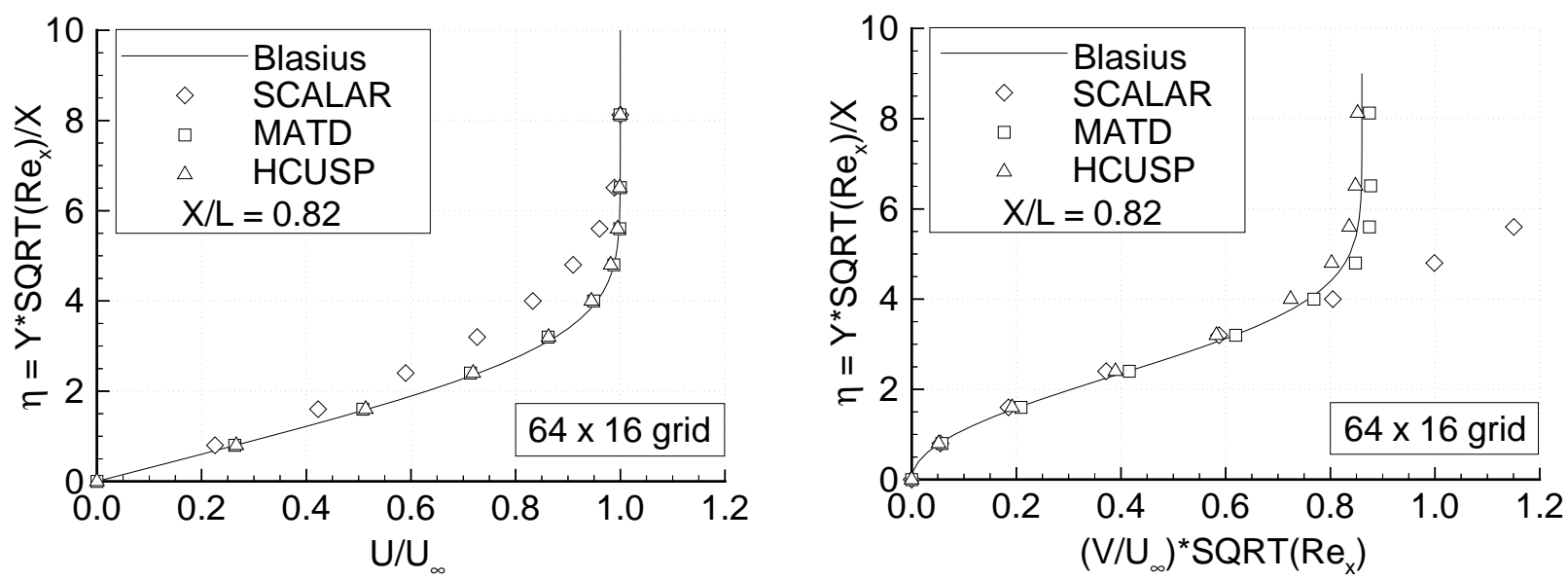

4a: Tangential and transverse velocity profiles, $\mathrm{X} / \mathrm{L}=0.82,64 \times 16$ grid.
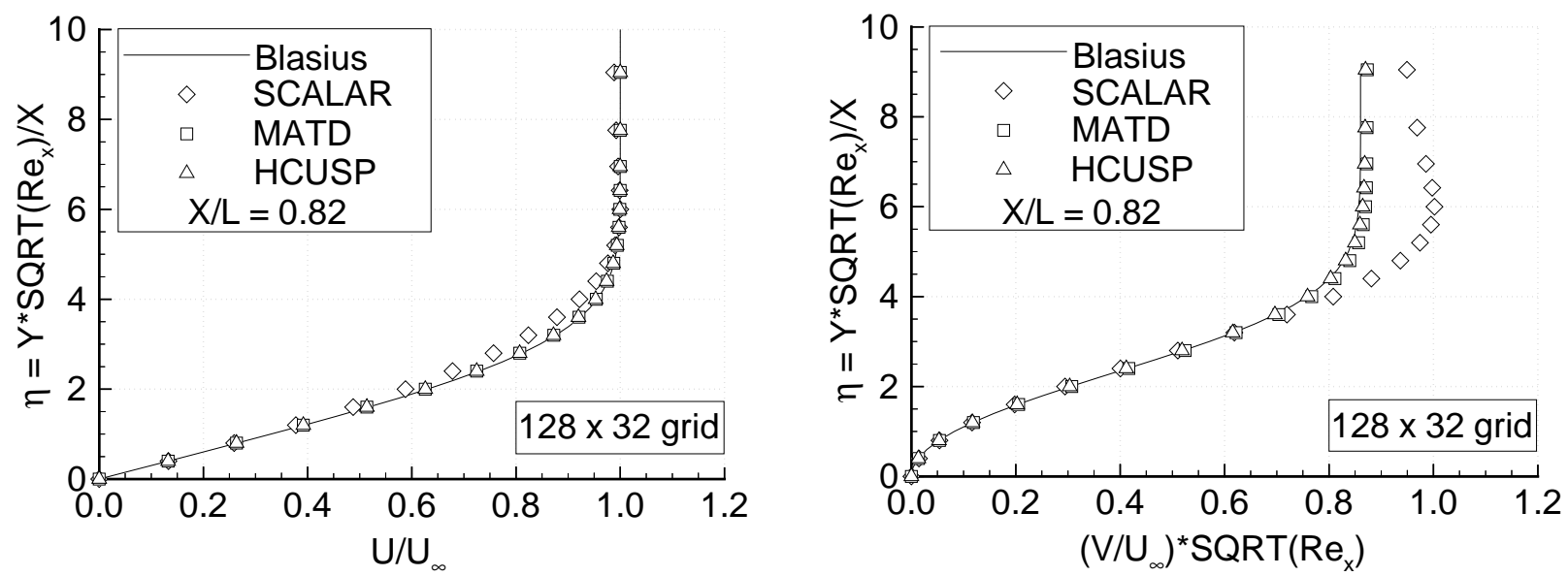

4b: Tangential and transverse velocity profiles, $\mathrm{X} / \mathrm{L}=0.82,128 \times 32$ grid.
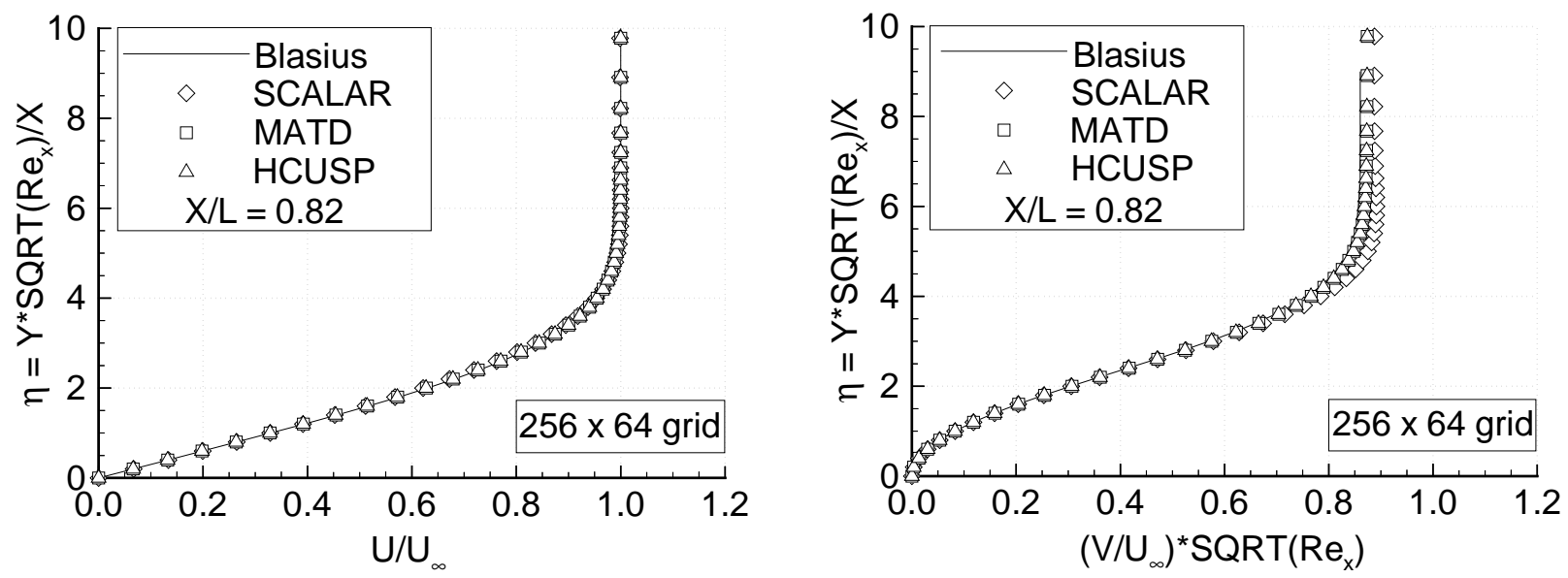

4c: Tangential and transverse velocity profiles, $\mathrm{X} / \mathrm{L}=0.82,256 \times 64$ grid.

Figure 4: Boundary-layer profiles on flat plate with $M=0.15$ and $R e=10^{5}$. 


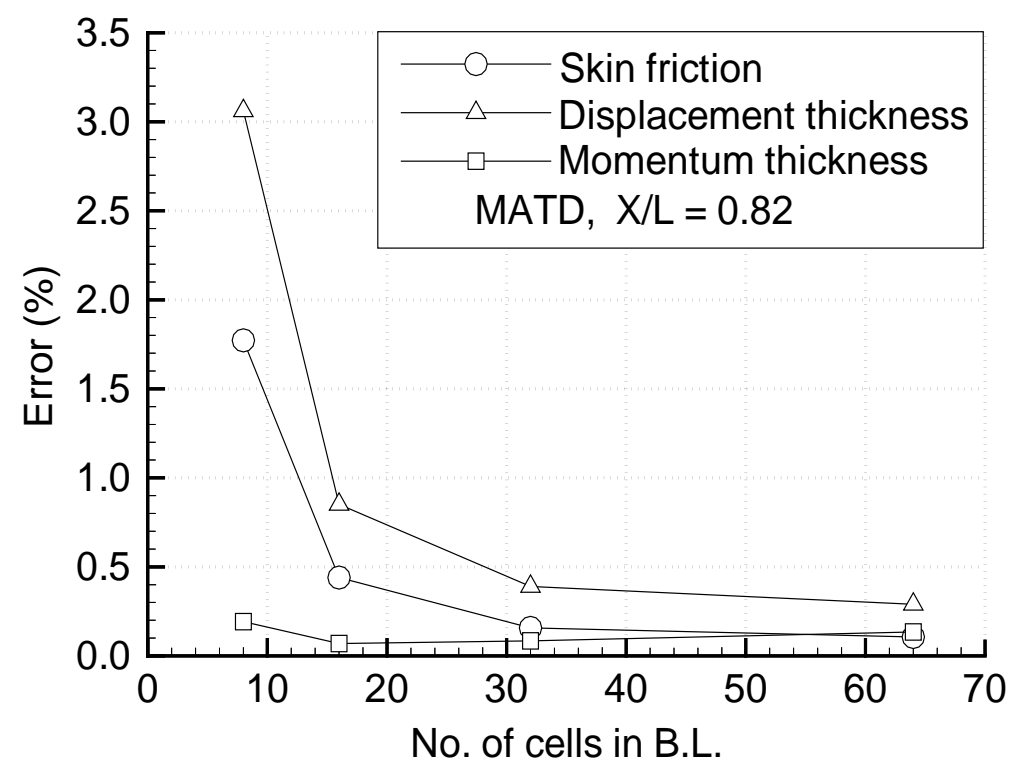

Figure 5a: Comparison of results with the MATD scheme and Blasius solution ( $M=0.15$ and $R e=10^{5}$ ).

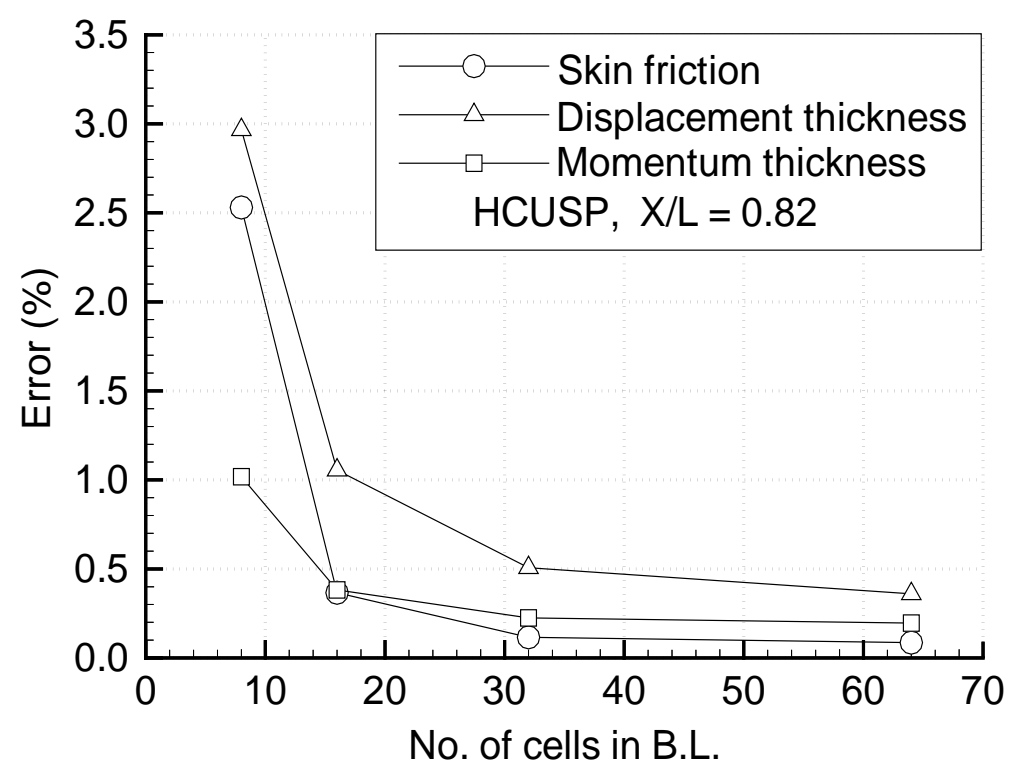

Figure 5b: Comparison of results with the HCUSP scheme and Blasius solution ( $M=0.15$ and $R e=10^{5}$ ). 


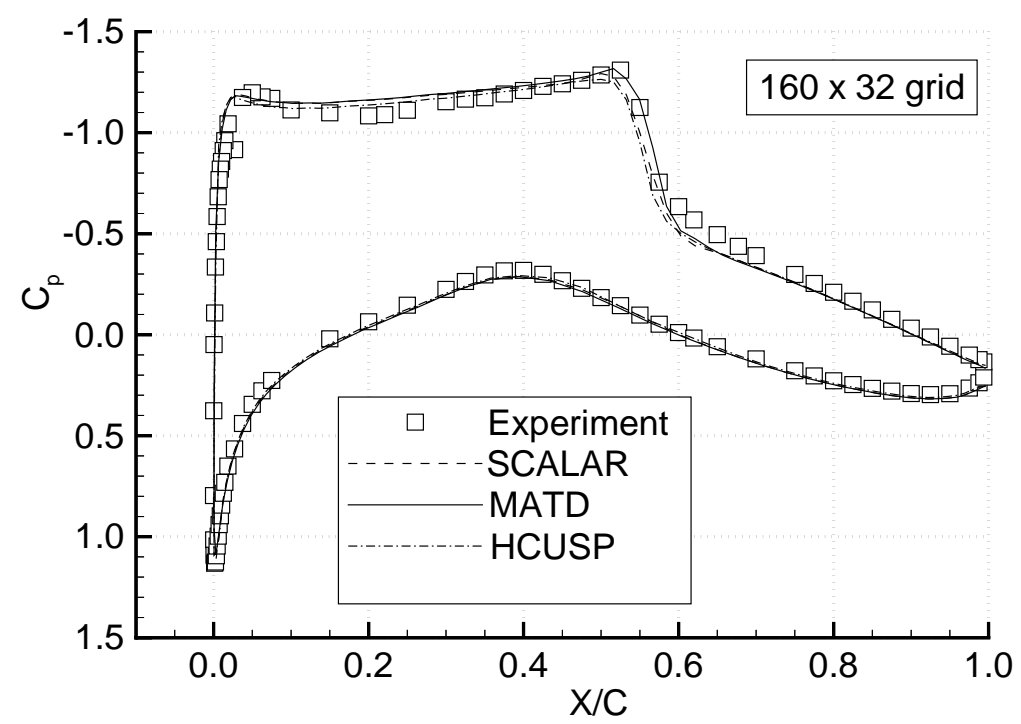

Figure 6a: Comparison of pressure distributions with SCALAR, MATD, and HCUSP schemes on $160 \times 32$ grid (RAE 2822 airfoil, $M=0.73, \alpha=2.79^{\circ}, R e=6.5 \times 10^{6}$ ).

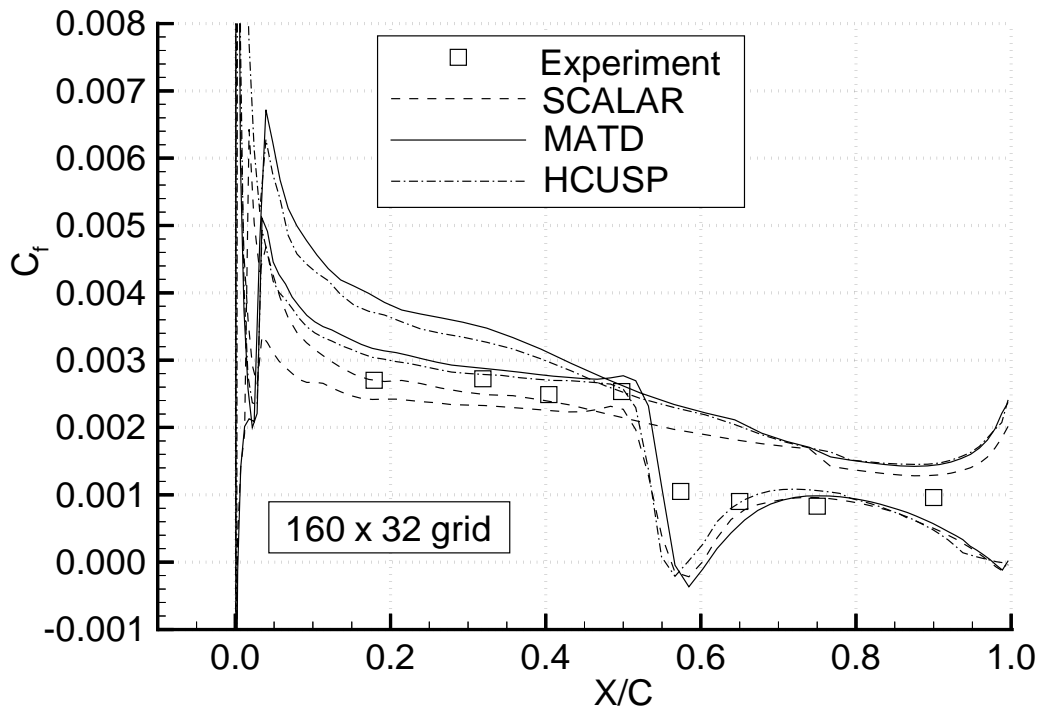

Figure 6b: Comparison of skin-friction distributions with SCALAR, MATD, and HCUSP schemes on $160 \times 32$ grid (RAE 2822 airfoil, $\left.M=0.73, \alpha=2.79^{\circ}, R e=6.5 \times 10^{6}\right)$. 


\title{
RAE 2822 airfoil
}

\author{
grid $160 \times 32$
}
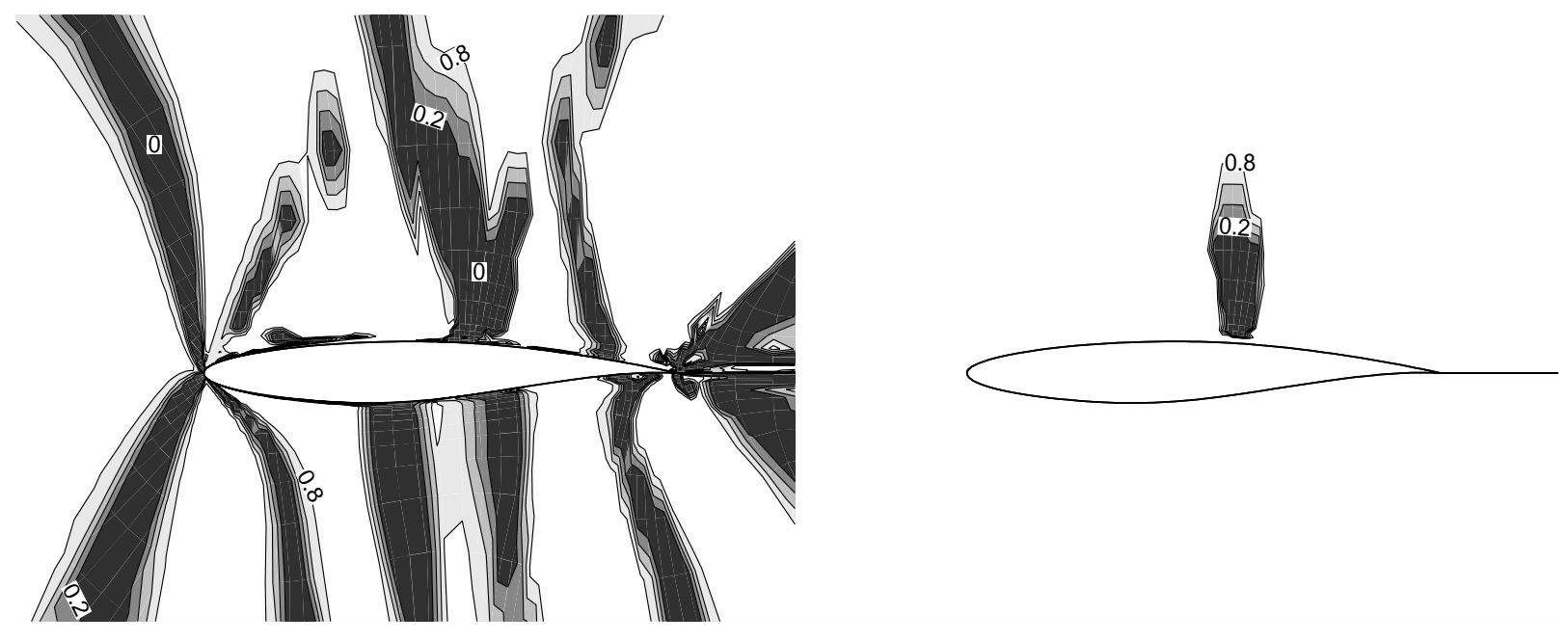

(a)

(b)

Figure 7: Contours of function $R(u, v)$ in limiter used with HCUSP scheme; (a) basic limiter and (b) modified limiter that depends on the contravariant Mach number and pressure switch. 
finest grid. In [24] the adverse effect of a smooth limiter on the accuracy of the solution in the vicinity of flow transition, and thus on the acceleration of the flow upstream of the shock, is demonstrated. Therefore, such a result with the HCUSP scheme could be a consequence of the smooth limiter being used. Thus, we examined the behavior of the limiter in the flow field.

The action of the limiter is revealed by the contour plot of Figure 7 for the minimum of the limiter function $R(u, v)$ (see (3.2)) taken over all four flow variables. The contours indicate that the basic limiter produces a first-order scheme over significant portions of the flow field. This result suggests that the inaccuracy on the coarse grid with the HCUSP scheme is not simply a consequence of the behavior of the limiter in the transition region. Figure 7 also shows contours of the modified function $R(u, v)$ which uses both the contravariant Mach number and the pressure switch of (2.13). With this function the low-order scheme occurs only at shock waves. Coarse grid results obtained with the basic and modified limiter functions are displayed in Figure 8. The shock locations computed with the modified HCUSP scheme and the MATD scheme are nearly the same.

In Figures 9 and 10 the solutions computed on the finer grids with the modified HCUSP scheme are compared with the other dissipation schemes. The pressure and skin-friction distributions obtained with the MATD and modified HCUSP schemes exhibit little difference on each mesh. The SCALAR scheme begins to show fairly close agreement with those from the other schemes only on the $640 \times 128$ grid. With both the SCALAR and the MATD schemes a nonphysical increase in the skin-friction solution on the upper surface appears at the trailing edge of the airfoil. This nonphysical increase is caused primarily by the aspect-ratio function of (2.11). As evident in Figure 11, this behavior does not occur in the solution obtained with the HCUSP scheme. The computed aerodynamic coefficients, including the pressure and friction contributions to the total drag, are given in Table II. On each mesh the lift and drag coefficients corresponding to the solution obtained with the MATD scheme exhibit the closest agreement with the $1280 \times 256$ grid values. There are only small discrepancies in the coefficients associated with the MATD and the modified HCUSP schemes on the $320 \times 64$ grid (see also Figure 12 ).

Convergence behavior for the HCUSP and MATD schemes is similar. For each scheme five levels of multigrid were used and either 50 or 100 cycles were executed on two coarser meshes in order to obtain an initial solution. On the $320 \times 64$ grid the average rate of reduction of the residual with both schemes is about 0.92 for 100 cycles on the finest mesh. Figure 13 shows the effect of modifying the limiter according to (3.8) and (3.2) on the convergence with the HCUSP scheme. It also indicates the effect of the modification given by (2.24) to $\nu c$ in the HCUSP scheme. The convergence is improved by using the $2-D$ formulation for the dissipation coefficient $\nu c$. Convergence stall can occur with the original limiter. With the modified limiter and the pressure switch this stall is prevented. Note that convergence with $\zeta=0$ was possible for this transonic case but not for the hypersonic case presented below.

The fourth case is the hypersonic 2-D flow over a blunt wedge. Figure 14 displays the second-order accurate solutions obtained for viscous and inviscid flow by using identical meshes of $64 \times 48$ cells. Physical diffusion is so large that the shock profile is significantly smeared in the viscous result. For inviscid flow, on the other hand, we obtain perfect capturing with a single interior point in the shock structure by using the formulation of 


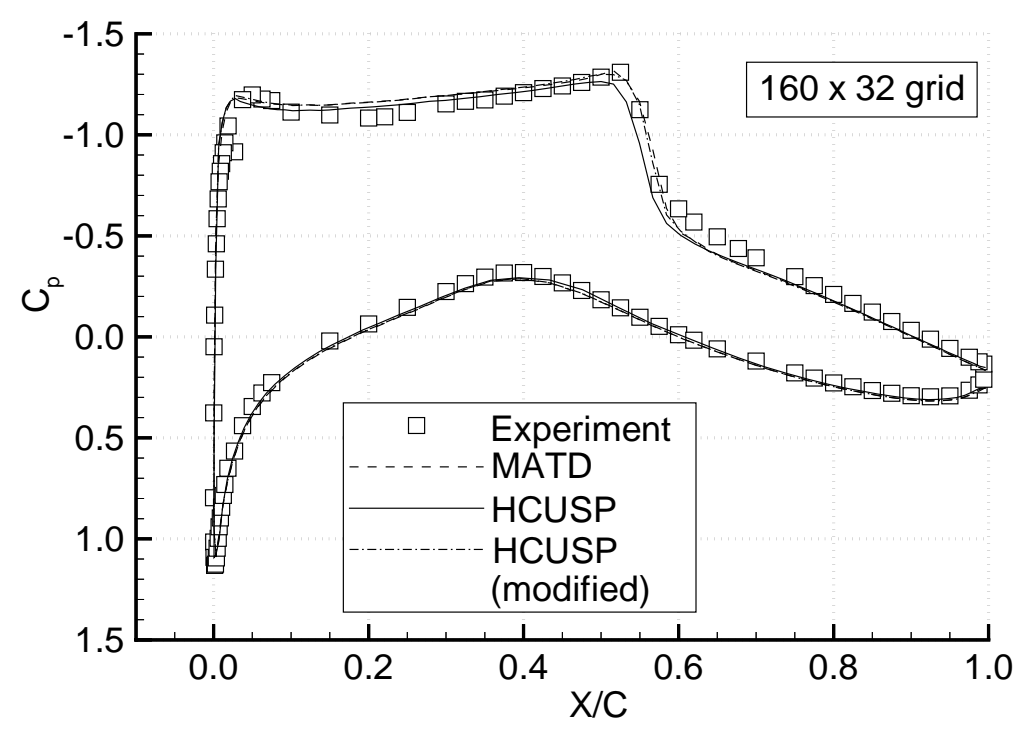

Figure 8a: Effect of modifications (pressure switch and reduced background dissipation) in HCUSP scheme on pressure $\left(160 \times 32\right.$ grid, RAE 2822 airfoil, $M=0.73, \alpha=2.79^{\circ}$, $\left.R e=6.5 \times 10^{6}\right)$.

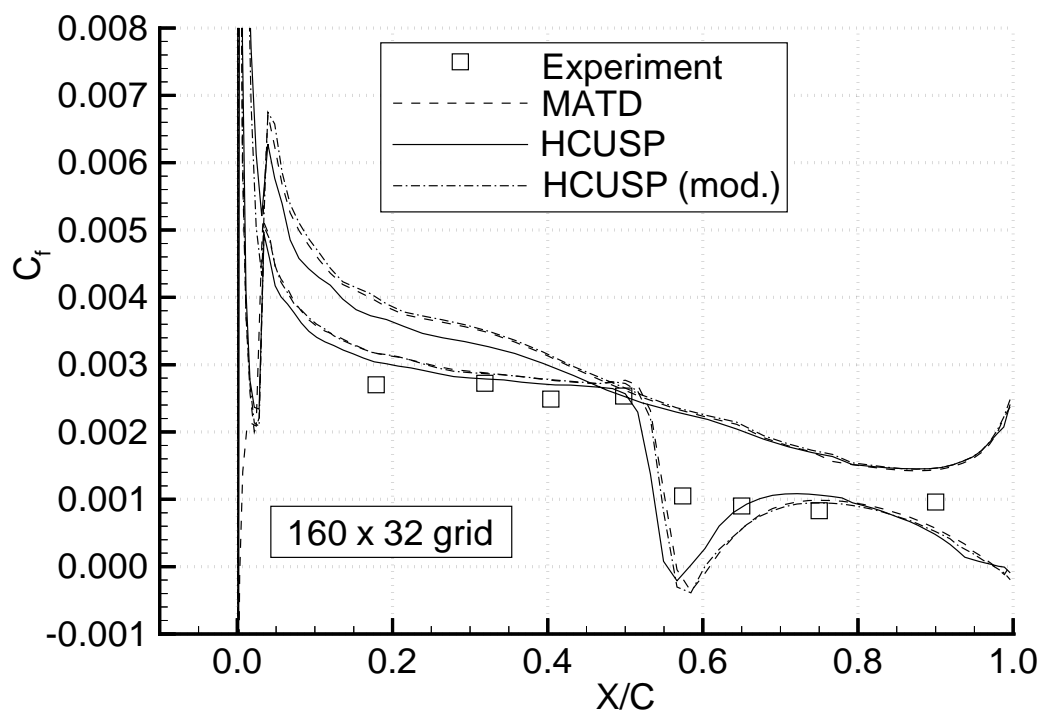

Figure 8b: Effect of modifications (pressure switch and reduced background dissipation) in HCUSP scheme on skin friction $\left(160 \times 32\right.$ grid, RAE 2822 airfoil, $M=0.73, \alpha=2.79^{\circ}$, $\left.R e=6.5 \times 10^{6}\right)$. 


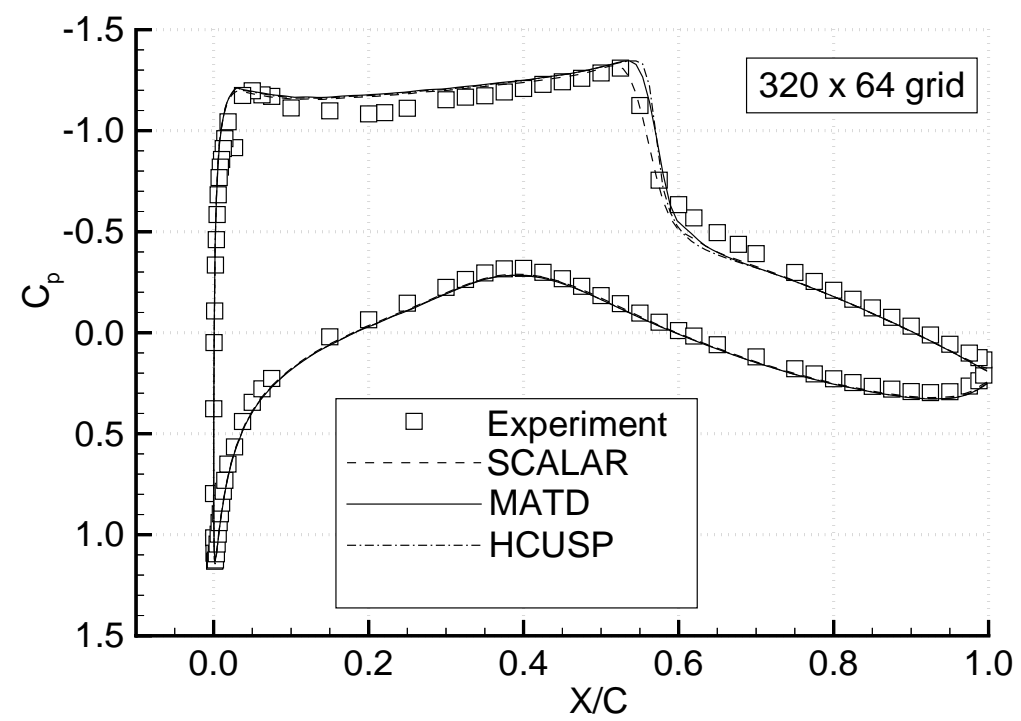

Figure 9a: Comparison of pressure distributions with SCALAR, MATD, and HCUSP schemes on $320 \times 64$ grid (RAE 2822 airfoil, $M=0.73, \alpha=2.79^{\circ}, R e=6.5 \times 10^{6}$ ).

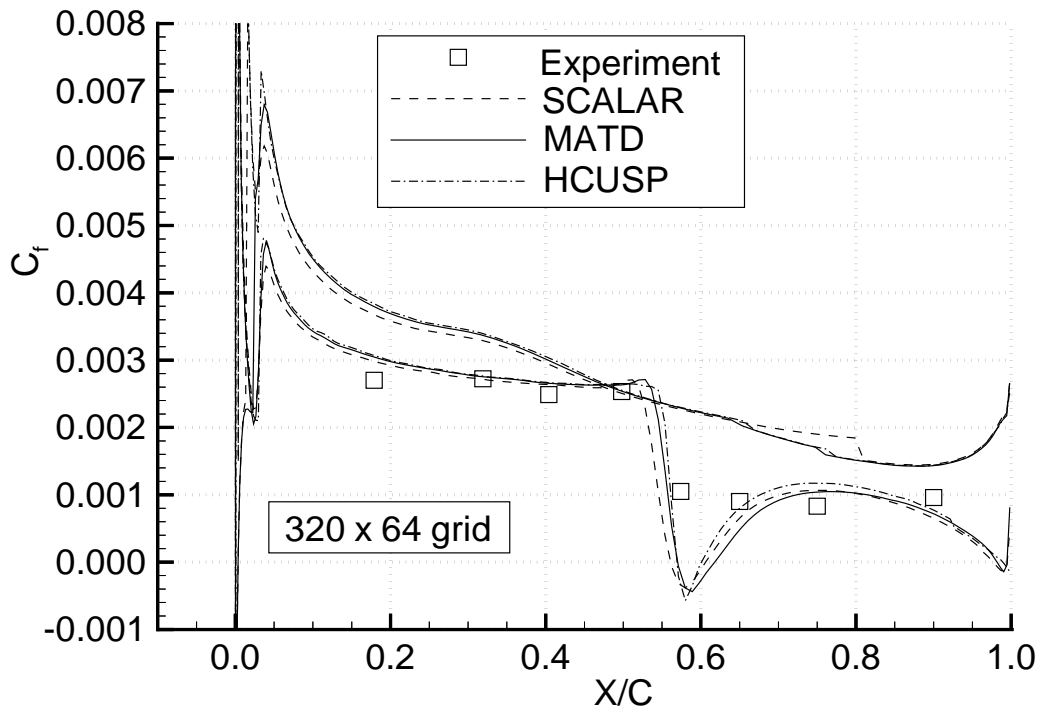

Figure 9b: Comparison of skin-friction distributions with SCALAR, MATD, and HCUSP schemes on $320 \times 64$ grid (RAE 2822 airfoil, $\left.M=0.73, \alpha=2.79^{\circ}, R e=6.5 \times 10^{6}\right)$. 


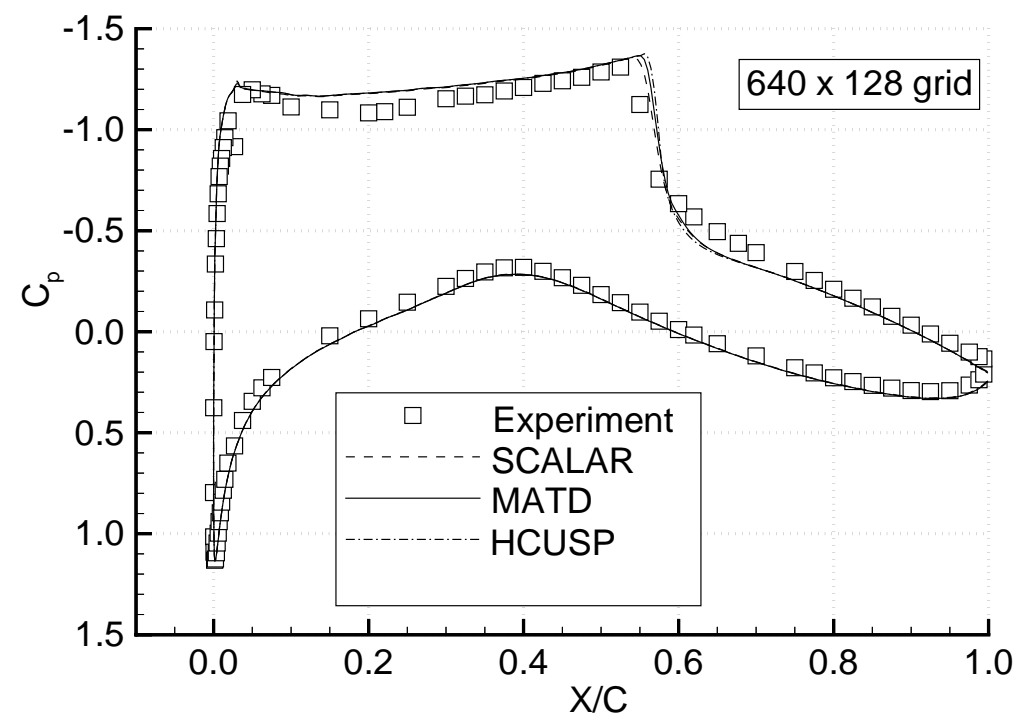

Figure 10a: Comparison of pressure distributions with SCALAR, MATD, and HCUSP schemes on $640 \times 128$ grid (RAE 2822 airfoil, $\left.M=0.73, \alpha=2.79^{\circ}, R e=6.5 \times 10^{6}\right)$.

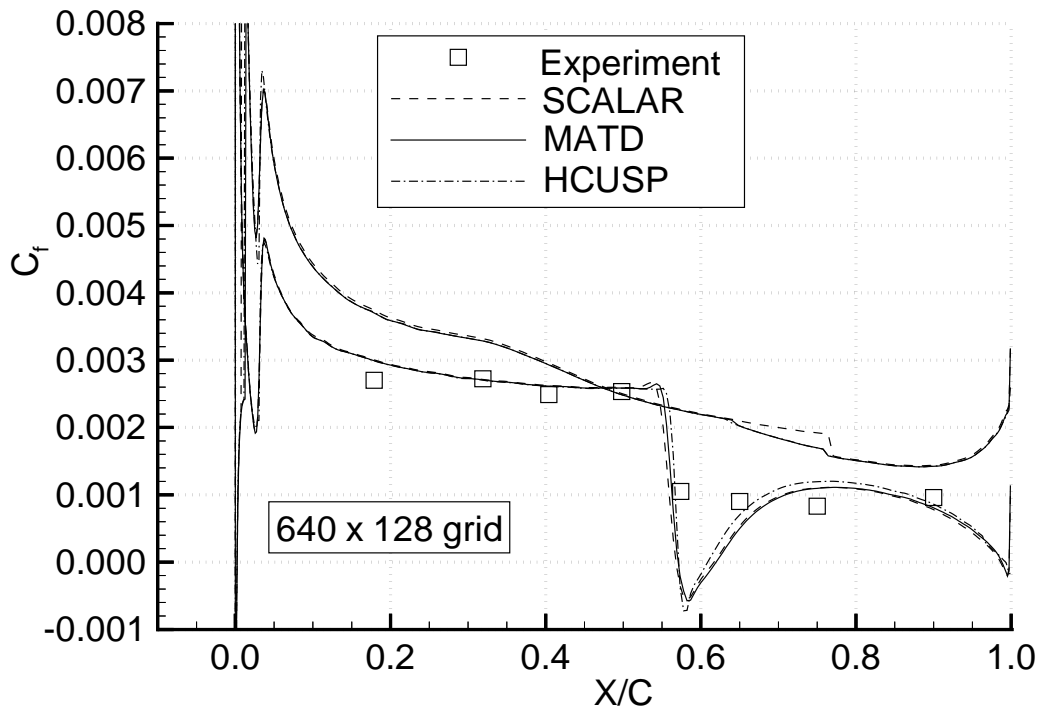

Figure 10b: Comparison of skin-friction distributions with SCALAR, MATD, and HCUSP schemes on $640 \times 128 \operatorname{grid}\left(\mathrm{RAE} 2822\right.$ airfoil, $\left.M=0.73, \alpha=2.79^{\circ}, R e=6.5 \times 10^{6}\right)$. 


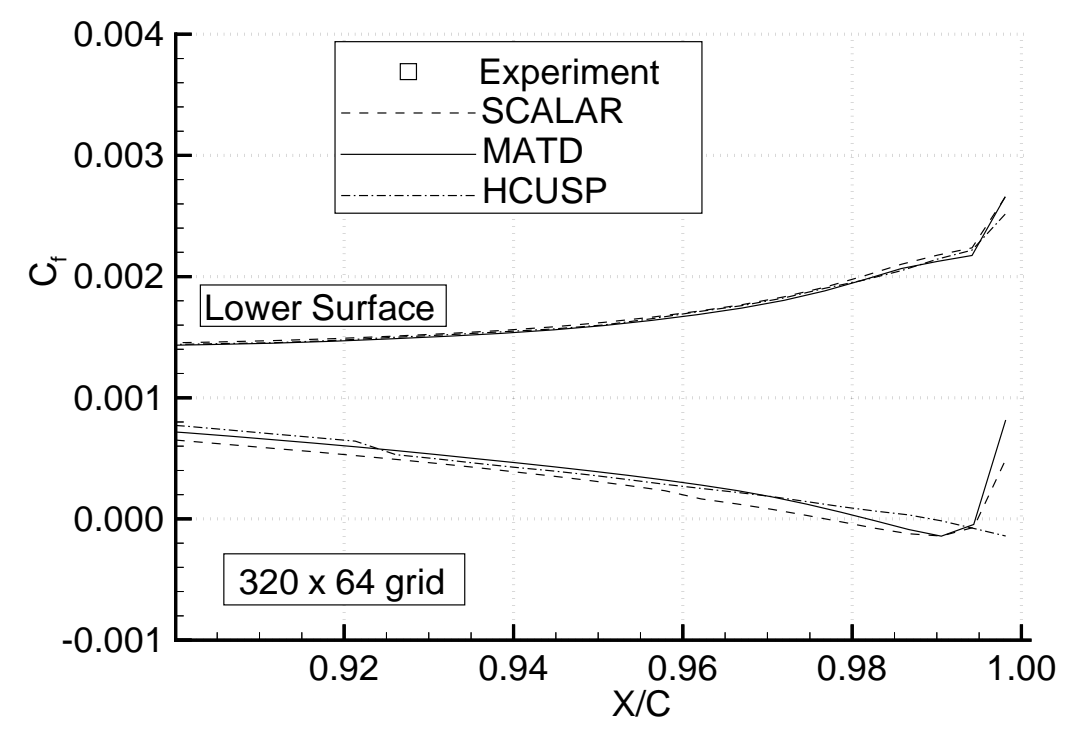

Figure 11: Behavior of skin-friction at airfoil trailing edge with SCALAR, MATD, and HCUSP schemes on $320 \times 64$ grid (RAE 2822 airfoil, $M=0.73, \alpha=2.79^{\circ}, R e=6.5 \times 10^{6}$ ).

\begin{tabular}{|c|r|c|c|c|c|}
\hline $\begin{array}{c}\text { Dissipation } \\
\text { Scheme }\end{array}$ & Grid & $C_{L}$ & $C_{D}$ & $C_{D p}$ & $C_{D f}$ \\
\hline SCALAR & $160 \times 32$ & 0.8172 & 0.01728 & 0.01275 & 0.004532 \\
& $320 \times 64$ & 0.8331 & 0.01743 & 0.01194 & 0.005487 \\
& $640 \times 128$ & 0.8532 & 0.01782 & 0.01225 & 0.005574 \\
\hline MATD & $160 \times 32$ & 0.8304 & 0.01818 & 0.01251 & 0.005662 \\
& $320 \times 64$ & 0.8538 & 0.01808 & 0.01250 & 0.005571 \\
& $640 \times 128$ & 0.8597 & 0.01799 & 0.01246 & 0.005535 \\
& $1280 \times 256$ & 0.8611 & 0.01800 & 0.01246 & 0.005544 \\
\hline HCUSP & $160 \times 32$ & 0.7987 & 0.01926 & 0.01367 & 0.005594 \\
& $320 \times 64$ & 0.8493 & 0.01831 & 0.01263 & 0.005679 \\
& $640 \times 128$ & 0.8592 & 0.01803 & 0.01245 & 0.005585 \\
\hline HCUSP & $160 \times 32$ & 0.8271 & 0.01760 & 0.01190 & 0.005701 \\
(modified) & $320 \times 64$ & 0.8565 & 0.01801 & 0.01234 & 0.005673 \\
& $640 \times 128$ & 0.8604 & 0.01798 & 0.01240 & 0.005581 \\
\hline
\end{tabular}

Table II: Lift and drag coefficients for turbulent flow over RAE 2822 airfoil $\left(M_{\infty}=0.73\right.$, $\left.\alpha=2.79^{\circ}, R e_{c}=6.5 \times 10^{6}\right)$. 


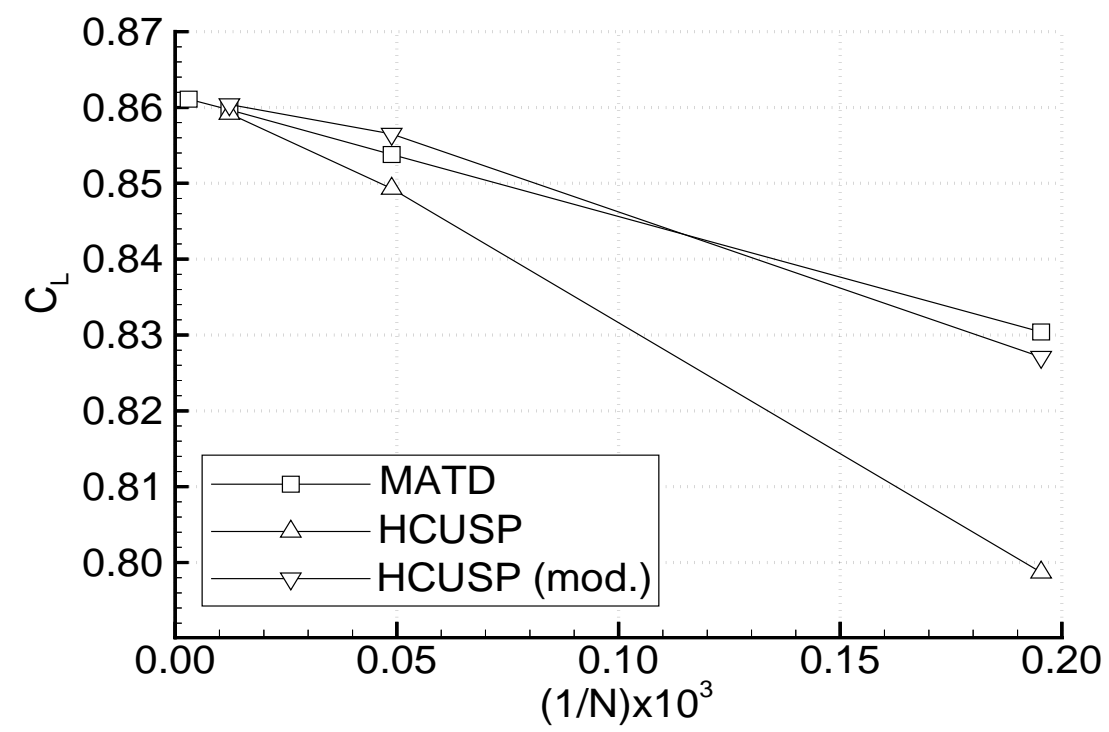

Figure 12a: Variation of lift coefficient with reciprocal of number of points (RAE 2822 airfoil, $\left.M=0.73, \alpha=2.79^{\circ}, \operatorname{Re}=6.5 \times 10^{6}\right)$.

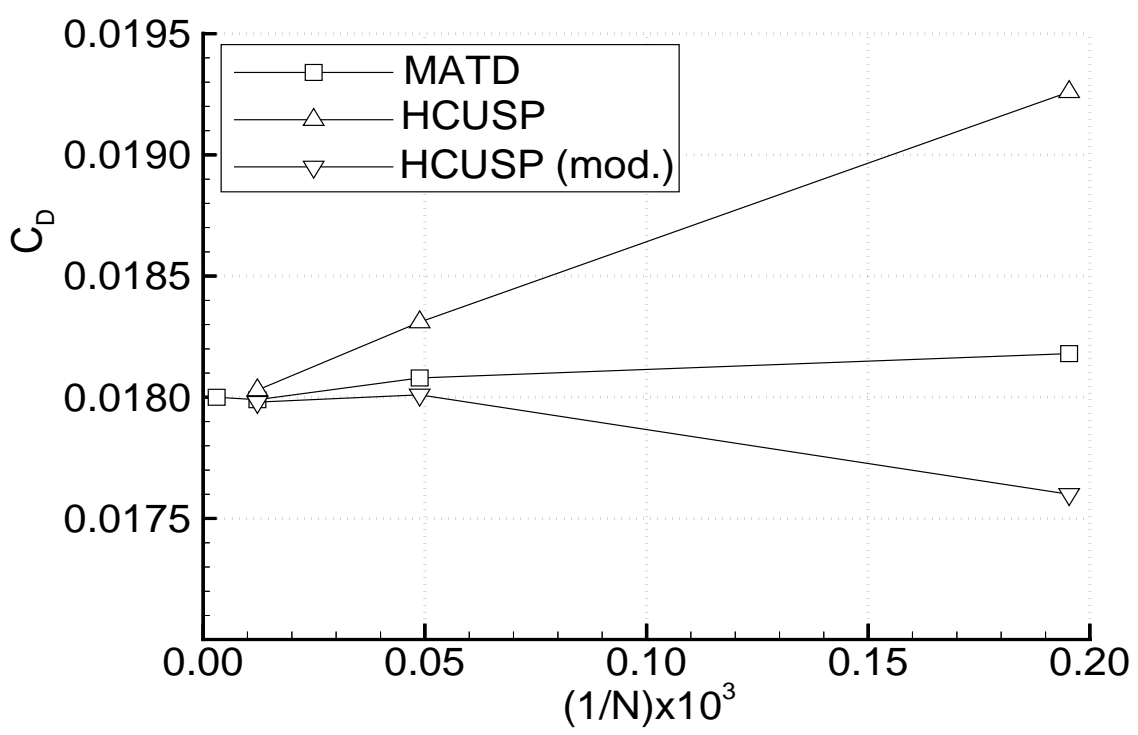

Figure 12b: Variation of drag coefficient with reciprocal of number of points (RAE 2822 airfoil, $M=0.73, \alpha=2.79^{\circ}, \operatorname{Re}=6.5 \times 10^{6}$ ). 


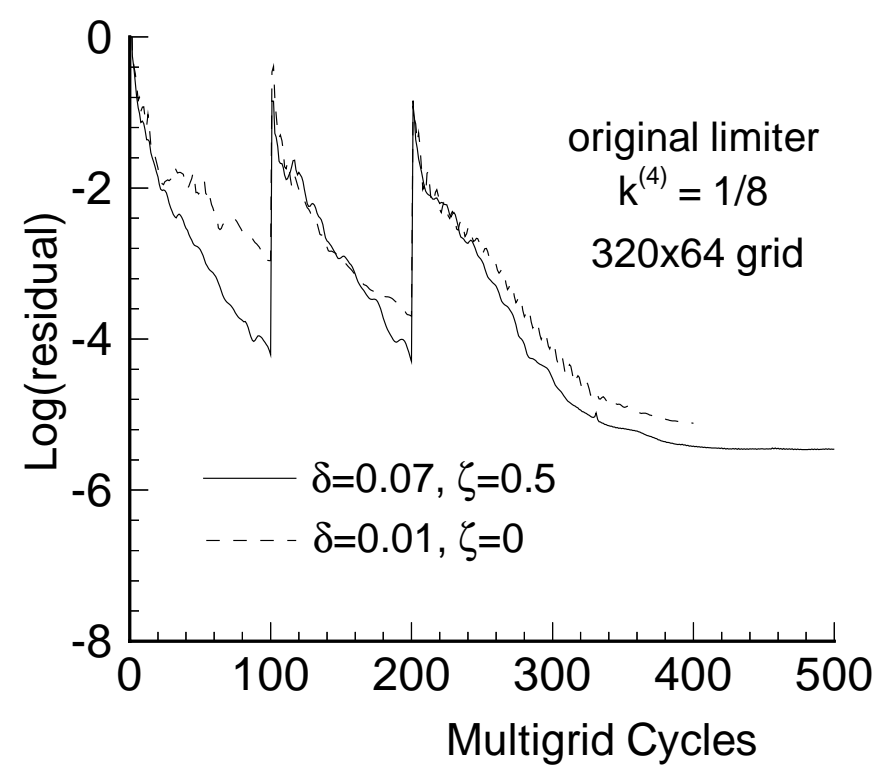

Figure 13a: Original limiter.

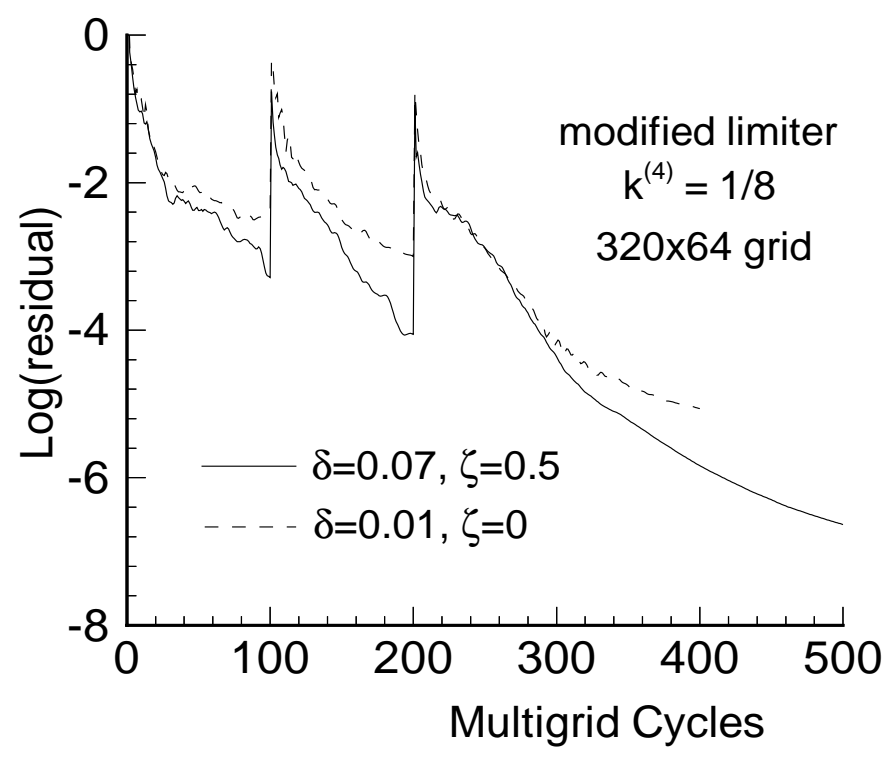

Figure 13b: Modified limiter.

Figure 13. Effect of limiter and modified $\nu c$ on convergence history of HCUSP scheme (RAE 2822 airfoil, $\left.M_{\infty}=0.73, \alpha=2.79^{\circ}, R e_{c}=6.5 \times 10^{6}\right)$. 

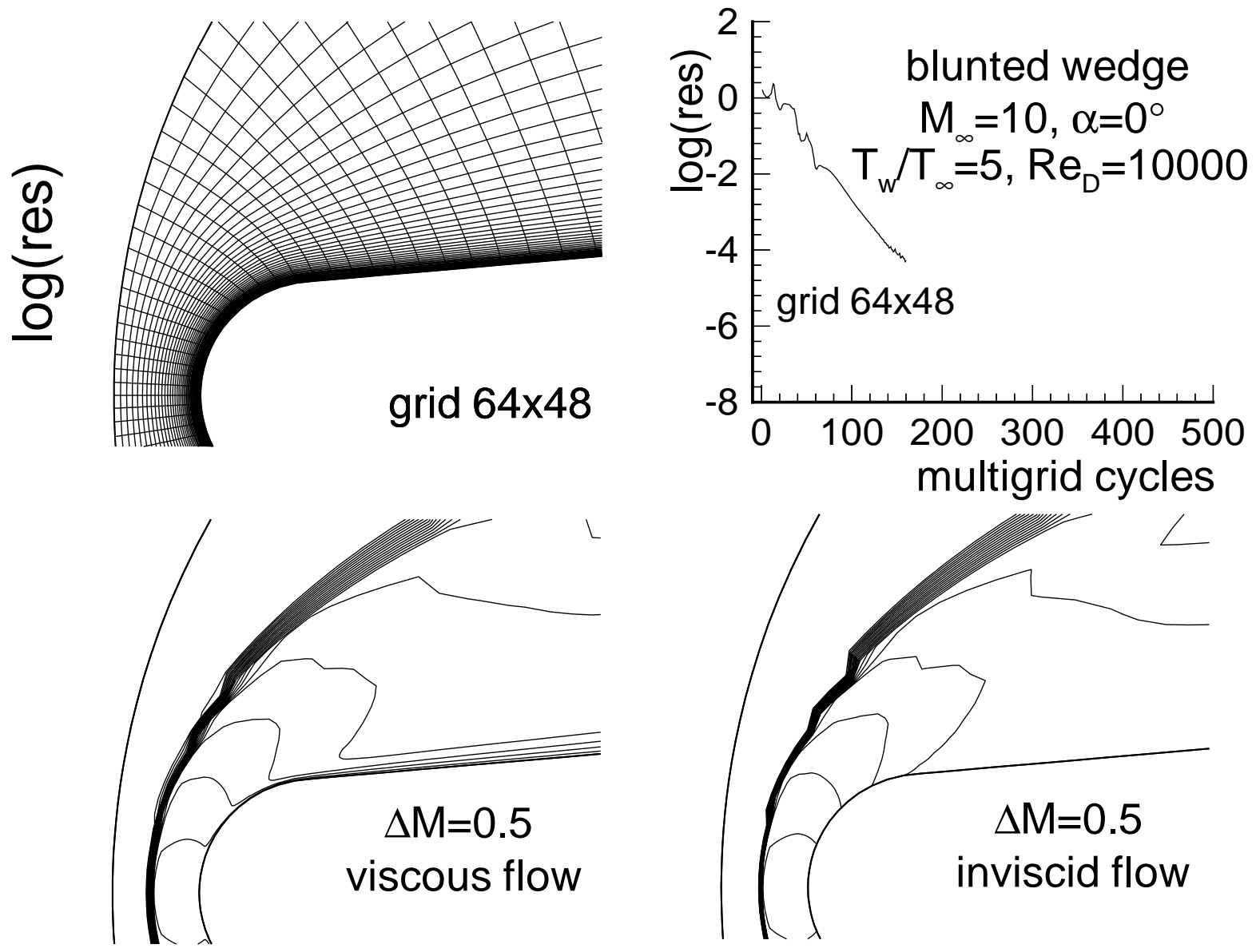

Figure 14: Viscous and inviscid hypersonic flow over 2-D wedge (second-order result). 
(2.23) and (2.24). Detailed comparisons of the hypersonic wedge flow solutions yielded by the CUSP scheme and AUSM have been presented in [16]. It was found that the shock capturing capabilities of both schemes are essentially equal. A comparison of shock profiles for the exact and the simplified coefficients is given in Figure 15. Here, we have chosen the first-order scheme in order to address the pure shock capturing capability of the CUSP scheme without interference from the limiter. The simplified dissipation coefficients of (2.26) produce strong oscillations at the shock, even though there is substantial physical diffusion present. Hence, it is concluded that an accurate implementation of dissipation coefficients is a requirement for hypersonic flows with strong shocks.

Some applications of the MATD scheme to hypersonic flow problems are given in [23]. However, we find that matrix dissipation combined with a pressure-based sensor in order to switch from second to fourth differences has not yet resulted in sufficient robustness to deal with hypersonic flow phenomena in general. In particular, it seems that the user defined coefficients in (2.13) - (2.15) need adjustment depending on the flow problem. Moreover, it is well known that matrix dissipation schemes suffer from an instability known as the carbuncle problem [15], and they need rather large values of $V_{n}$ and $V_{l}$ in order to restore stability.

The final set of results show the behavior of the HCUSP scheme with preconditioning. Inviscid solutions for flow over a NACA 0012 airfoil were computed on a C-type grid with $224 \times 40$ cells and clustering at the leading and trailing edges. In Figure 16 Mach number contours delineate the effect of the free-stream Mach number on the solutions obtained with the preconditioned HCUSP scheme. Figure 17 clearly illustrates the benefits of preconditioning on the HCUSP scheme. There is a substantial improvement in not only the quality of the solution but also the convergence behavior with the scheme.

Comparisons of computation times indicate that the HCUSP scheme needs about $25 \%$ more computer time than the basic scalar dissipation of Section 2.1. The MATD scheme only requires about $15 \%$ additional time. This reduction is primarily a consequence of the single evaluation of the limiter function. Due to lower inherent dissipation, computations with the HCUSP formulation converge somewhat slower for transonic flows than those with simple scalar dissipation. The major advantage of the HCUSP approach is that it is more accurate and more robust than scalar viscosity. Our numerical tests indicate that the accuracy of the CUSP scheme is close that of matrix dissipation for transonic flows provided the firstorder scheme is activated at shock waves only. For hypersonic flows it seems to be more robust than the matrix viscosity even though it is not positive. Since the HCUSP scheme is implemented through artificial dissipative terms, it does not have to be applied at each stage of the Runge-Kutta method. In particular, the diffusive fluxes can be evaluated only at the first, third, and fifth stages of a five-stage method, as is typically done for the scalar dissipation. 

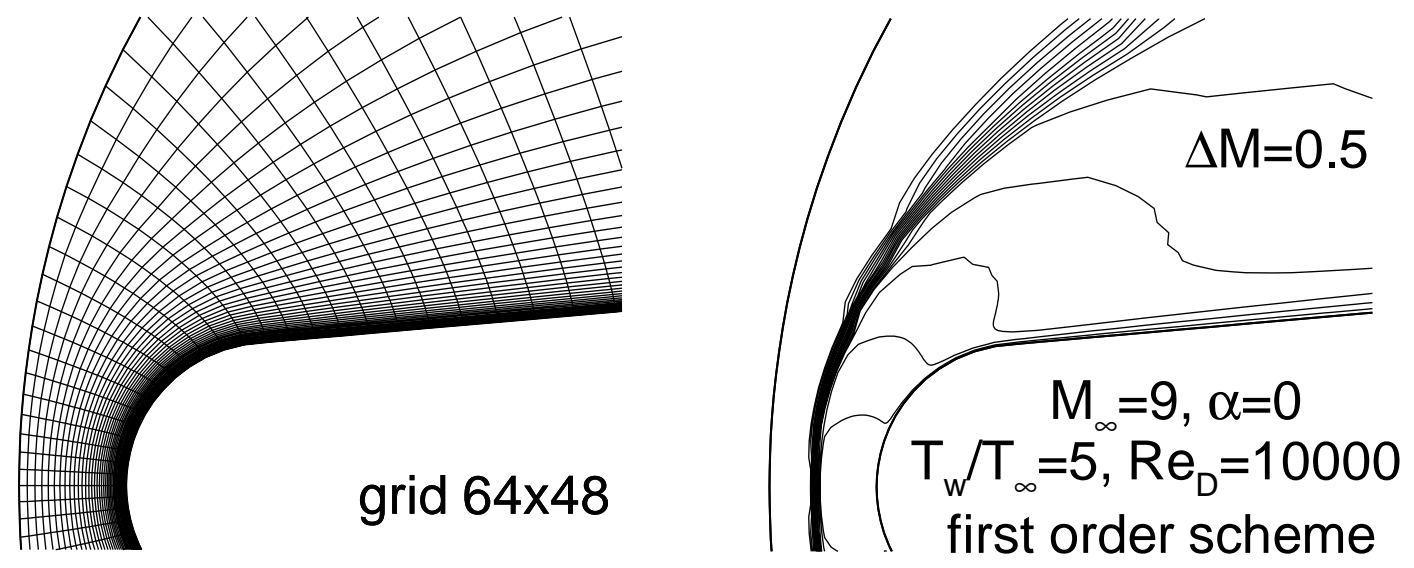

center line flow

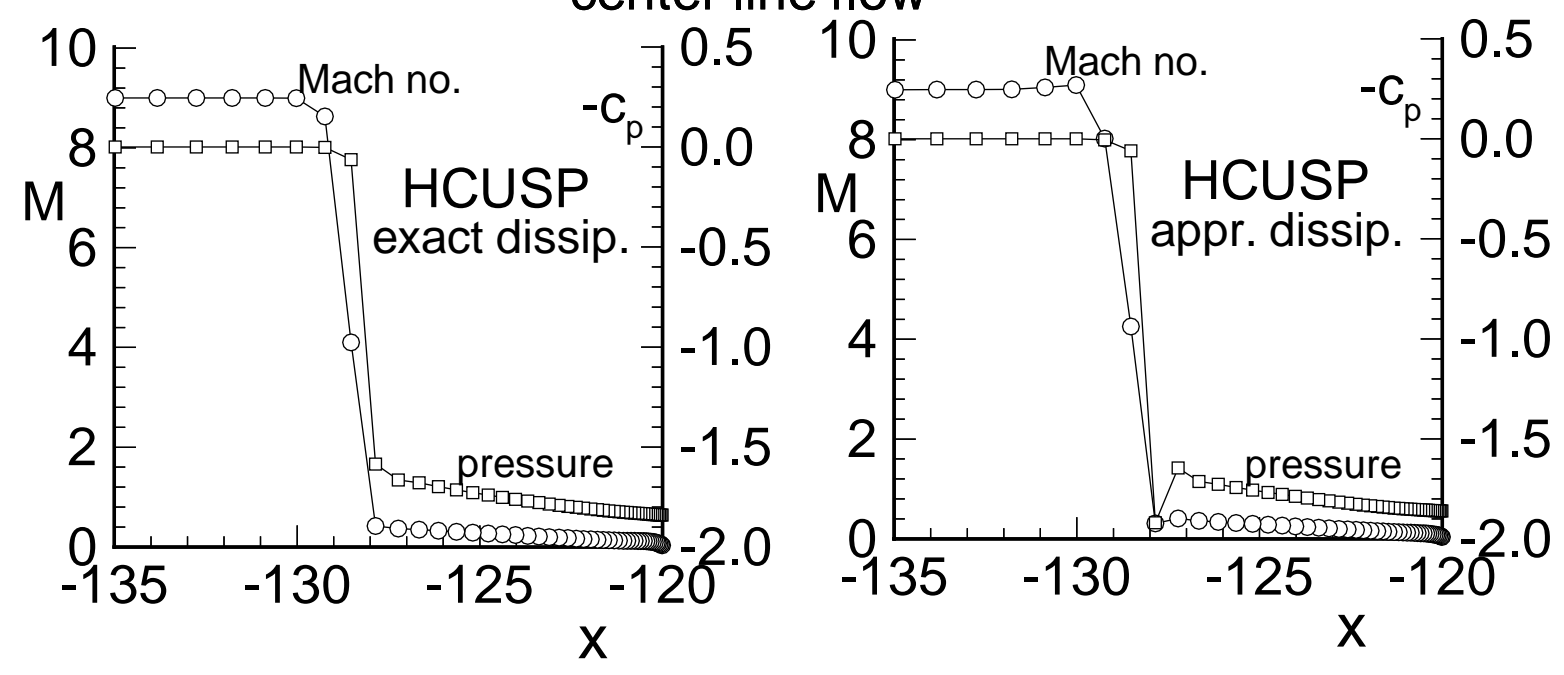

Figure 15: Influence of HCUSP dissipation coefficients on hypersonic flow over 2-D wedge. 

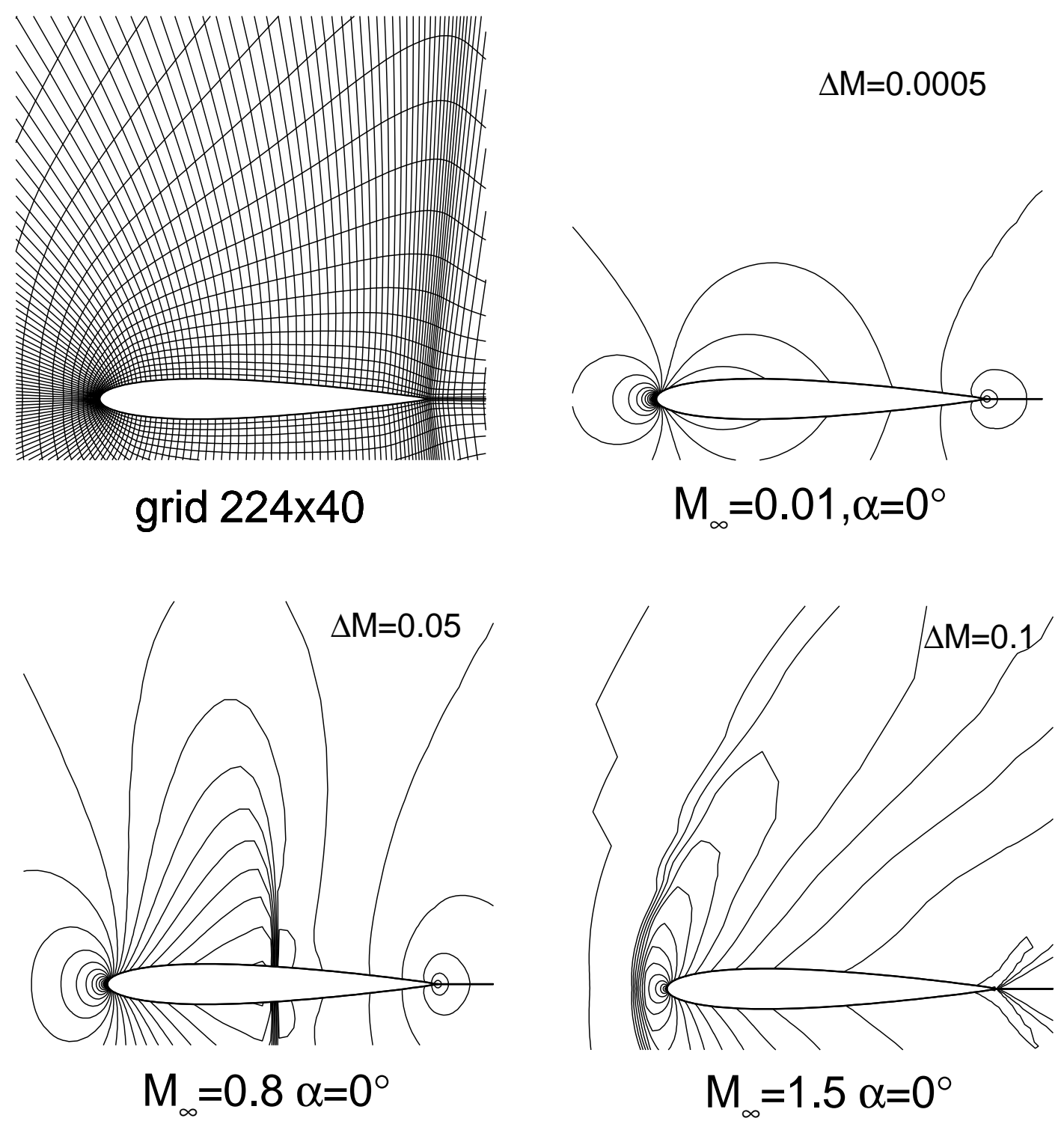

Figure 16: Influence of free-stream Mach number on the inviscid flow around NACA 0012 airfoil with the preconditioned HCUSP scheme. 

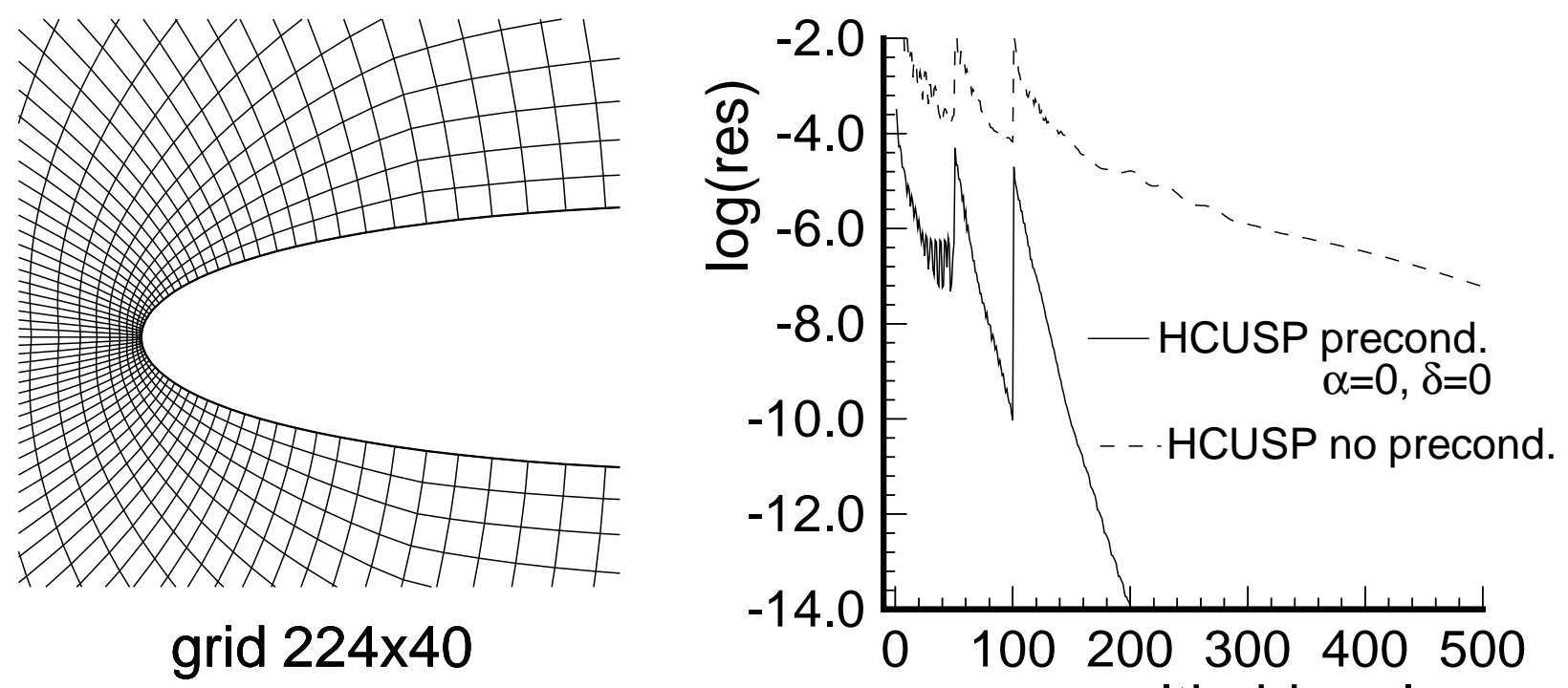

\section{grid $224 \times 40$}

\section{$M_{\infty}=0.05, \alpha=0^{\circ} \quad$ multigrid cycles}
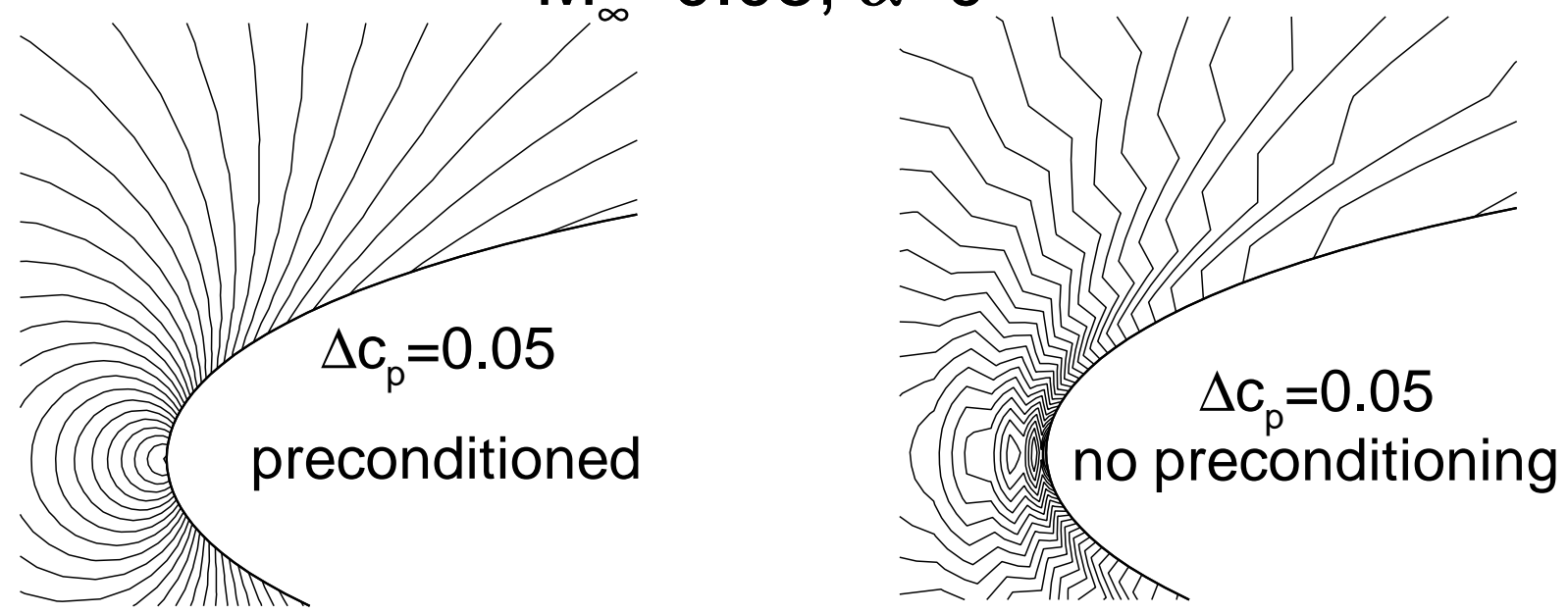

Figure 17: Influence of preconditioning on the HCUSP scheme. 


\section{Concluding Remarks}

The CUSP scheme has been studied and analyzed. A detail comparison has been made between the CUSP, MATD, and scalar dissipation schemes. For transonic inviscid flows the CUSP scheme allows better resolution of shock waves, since they are captured with one interior point. However, the aerodynamic quantities such as lift and drag obtained with the original CUSP scheme are not as accurate on coarser meshes (i.e., $320 \times 64$ cells or less) as those calculated with the MATD scheme. Both the CUSP and MATD formulations can give high accuracy in the computation of viscous flows. In the case of high Re number flow over a flat plate, each of these schemes required only eight points in the boundary layer to have errors in computed skin-friction, displacement thickness, and momentum thickness that do not exceed $3 \%$. Four times as many points is necessary to obtain comparable accuracy with the scalar scheme. For transonic viscous flows and coarser meshes the accuracy in aerodynamic coefficients is somewhat better with the MATD scheme than with the original CUSP scheme. This loss in accuracy with the CUSP scheme on coarser grids appears to be a consequence of the limiter producing a first-order scheme over significant portions of the flow field and higher levels of background dissipation.

Modifications to the CUSP scheme for improving the coarse-grid accuracy have been presented. These changes restrict the activation regions of the first-order scheme to the neighborhoods of shock waves according to (3.2) and reduce background dissipation using the limiter of (3.8). They allow the CUSP scheme to give comparable accuracy to that obtained with the MATD scheme on coarse meshes. With these modifications to the CUSP scheme, convergence stall has been removed. Convergence has been further improved by introducing the aspect-ratio scaling factor of (2.24).

In comparison to the scalar scheme the CUSP scheme requires roughly $25 \%$ more computer time while the MATD scheme needs about $15 \%$ more time. In general, convergence behavior with the CUSP and MATD schemes is similar.

With our present choice of HCUSP dissipation coefficients it has been shown that the resolution of strong shock waves occurring in hypersonic flows is possible whereas the simplified coefficients that were published previously failed. At this point the HCUSP scheme appears to be a better choice than the present MATD scheme for hypersonic flow problems.

\section{Acknowledgment}

We would like to thank Dr. Norbert Kroll for his help. 


\section{References}

[1] S. Allmaras, Contamination of laminar boundary layers by artificial dissipation in Navier-Stokes solutions, in Proceedings, Conference on Numerical Methods in Fluid Dynamics, Reading, England, UK, April 1992.

[2] P. H. Cook, M. A. McDonald, and M. C. P. Firmin, AERFOIL RAE 2822 pressure distributions, and boundary layer and wake measurements, AGARD Advisory Report 138, 1979 (unpublished).

[3] A. Harten, High resolution schemes for hyperbolic conservation laws, J. Comput. Phys. 49,357(1983).

[4] A. Jameson, W. Schmidt, and E. Turkel, Numerical solutions of the Euler equations by finite volume methods using Runge-Kutta time-stepping schemes, AIAA Paper 811259, 1981 (unpublished).

[5] A. Jameson, Artificial diffusion, upwind biasing, limiters and their effect on accuracy and multigrid convergence in transonic and hypersonic flow, AIAA Paper 93-3559, 1993 (unpublished).

[6] A. Jameson, Analysis and design of numerical schemes for gas dynamics I: artificial diffusion, upwind biasing, limiters and their effect on accuracy and multigrid convergence, Int. J. Comput. Fluid Dyna. 4,171(1995).

[7] A. Jameson, Analysis and design of numerical schemes for gas dynamics II: artificial diffusion and discrete shock structure, Int. J. Comput. Fluid Dyna. 5,1(1995).

[8] A. Jameson, Positive schemes and shock modelling for compressible flows, Int. J. Numer. Meth. Eng. 20,743(1995).

[9] P. Jorgenson and E. Turkel, Central difference TVD schemes for time dependent and steady state problems, J. Comput. Phys. 107,297(1993).

[10] M.-S. Liou and C. J. Steffen, A new flux splitting scheme, J. Comput. Phys. 107,23(1993).

[11] M.-S. Liou, A sequel to AUSM: AUSM+, J. Comput. Phys. 129,364(1996).

[12] M.-S. Liou and Y. Wada, A quest towards ultimate numerical flux schemes, CFD Review, edited by M. M. Hafez and K. Oshima, (John Wiley and Sons, 1995), p. 251.

[13] L. Martinelli and A. Jameson, Validation of a multigrid method for the Reynolds averaged equations, AIAA Paper 88-0414, 1988 (unpublished).

[14] T. H. Pulliam, Artificial dissipation for the Euler equations, AIAA J. 24,1931(1986).

[15] J. J. Quirk, A contribution to the great Riemann solver debate, Int. J. Numer. Meth. in Fluids 18,555(1994). 
[16] R. Radespiel, J. M. A. Longo, S. Bruck, and D. Schwamborn, Efficient numerical simulation of complex 3D flows with large contrast, AGARD-CP-578, 1996.

[17] R. Radespiel and N. Kroll, Accurate flux vector splitting for shocks and shear layers, J. Comput. Phys. 121,66(1995).

[18] R. Radespiel and R. C. Swanson, Progress with multigrid schemes for hypersonic flow problems, J. Comput. Phys. 116,103(1995).

[19] P. L. Roe, Approximate Riemann solvers, parameter vectors and difference schemes, J. Comput. Phys. 43,357(1981).

[20] H. Schlichting, Boundary Layer Theory, VII-th edition, McGraw-Hill, New York, 1979.

[21] R. C. Swanson and E. Turkel, Artificial dissipation and central difference schemes for the Euler and Navier-Stokes equations, in AIAA 8th Computational Fluid Dynamics Conference, AIAA, 1987 (AIAA Paper 87-1107-CP), p. 55.

[22] R. C. Swanson and E. Turkel, On central difference and upwind schemes, J. Comput. Phys. 101,292(1992).

[23] R. C. Swanson, E. Turkel, and J. A. White, An effective multigrid method for highspeed flows, Comm. Appl. Numer. Meth. 8,671(1992)

[24] R. C. Swanson and E. Turkel, Aspects of a high-resolution scheme for the NavierStokes equations, in AIAA 11th Computational Fluid Dynamics Conference, AIAA, 1993 (AIAA Paper 93-3372-CP), p. 805.

[25] R. C. Swanson, R. Radespiel, and E. Turkel, Comparison of several dissipation algorithms for central difference schemes, in AIAA 13th Computational Fluid Dynamics Conference, AIAA, 1997 (AIAA Paper 97-1945-CP), p. 580.

[26] E. Tadmor, Convenient total variation diminishing conditions for nonlinear difference schemes, SIAM J. Numer. Anal. 25,1002(1988).

[27] S. Tatsumi, L. Martinelli, A. Jameson, A design, implementation and validation of flux limited schemes for the solution of the compressible Navier-Stokes equations, AIAA Paper 94-0647, 1994 (unpublished).

[28] S. Tatsumi, L. Martinelli, and A. Jameson, A new high resolution scheme for compressible viscous flow with shocks, AIAA Paper 95-0466, 1995 (unpublished).

[29] S. Tatsumi, L. Martinelli, and A. Jameson, Flux-limited schemes for the compressible Navier-Stokes equations, AIAA J. 33,252(1995).

[30] E. Turkel, A review of preconditioning methods for fluid dynamics, Appl. Numer. Math. 12,257(1993).

[31] E. Turkel, R. Radespiel, and N. Kroll, Assessment of two preconditioning methods for aerodynamic problems, Comput. \& Fluids 26,613(1997). 
[32] E. Turkel, V. N. Vatsa, and R. Radespiel, Preconditioning methods for low speed flow, in 14th AIAA Applied Aerodynamics Conference, AIAA, 1996 (AIAA Paper 96-2460CP), p. 650 .

[33] D. L. Tweedt, R. V. Chima, and E. Turkel, Preconditioning for numerical simulation of low Mach number three-dimensional viscous turbomachinery flows, AIAA Paper 97-1828, 1997 (unpublished).

[34] B. van Leer, Flux vector splitting for the Euler equations, Eighth Int. Confer. on Numerical Methods in Fluid Dynamics, 1982, edited by Krause, Lect. Notes in Phys., (Springer-Verlag, New York/Berlin, 1982), No. 170, p. 507.

[35] Y. Wada and M.-S. Liou, An accurate and robust flux splitting scheme for shock and contact discontinuities, SIAM J. Sci. Comput. 18 (1997). 